\title{
DISTINGUISHED BASES AND MONODROMY OF COMPLEX HYPERSURFACE SINGULARITIES
}

\author{
WOLFGANG EBELING
}

\begin{abstract}
We give a survey on some aspects of the topological investigation of isolated singularities of complex hypersurfaces by means of Picard-Lefschetz theory. We focus on the concept of distinguished bases of vanishing cycles and the concept of monodromy.
\end{abstract}

\section{Contents}

Introduction 1

1. Distinguished bases of vanishing cycles 3

2. Coxeter-Dynkin diagram and Seifert form 11

3. Change of basis 13

4. Computation of intersection matrices 18

5. The discriminant and the Lyashko-Looijenga map 21

6. Special singularities 24

7. The monodromy group 31

8. Topological equivalence $\quad 35$

References $\quad 37$

Index $\quad 46$

\section{INTRODUCTION}

The pioneering fibration theorem of J. Milnor [Mi68a] opened the way to study the topology of isolated complex hypersurface singularities. To study the topology of real smooth manifolds one can use Morse theory. The idea of Morse theory is that the topological type of the level set of a real function changes when passing through a critical value. In order to study the topology of the singularity defined by a complex analytic function one can investigate the level sets of this function. The complex analogue of Morse theory is Picard-Lefschetz

2010 Mathematics Subject Classification. 14D05, 32S50, 58K10.

Partially supported by DFG. 
theory. It is older than Morse theory and goes back to E. Picard and S. Simart [PS97] and to S. Lefschetz [Lef24].

Around 1967-1969, the Picard-Lefschetz theory experienced a revival when it was brought into an algebraic form by A. Grothendieck, P. Deligne, and N. Katz in [DK73]. On a more modest scale, the theory was applied in the late 1960s and early 1970s to the analysis of isolated singularities of complex hypersurfaces. The first fundamental contributions were made by F. Pham [Ph65], Lê Dũng Tráng [Le73, Le78], E. Brieskorn [Br70, Appendix], K. Lamotke [Lam75], and A. M. Gabrielov [Ga73, Ga74a, Ga74b, Ga79]. Gabrielov coined the notion of "distinguished bases". Instead of passing through a critical value, the fundamental principle of Picard-Lefschetz theory is going around a critical value in the complex plane. Roughly speaking, to the critical values there corresponds a distinguished basis of vanishing cycles and the change of the topology of the level set is given by the "monodromy". This article is a survey of these fundamental concepts and the further developments.

Nowadays, there are good references for this subject. There is a survey article by S. M. Gusein-Zade [GZ77] and a later one by Brieskorn [Br88]. A very good reference is the second volume of the book of V. I. Arnold, Gusein-Zade, and A. Varchenko [AGV88]. The book of E. Looijenga [Loo84] is devoted to isolated complete intersection singularities, but it also contains relevant information about hypersurface singularities which are a special case. Moreover, there are also textbooks by D. Bättig and H. Knörrer [BK91] (in German) and by the author [Eb07]. The author has already written a survey on the classical monodromy [Eb06]. We keep the intersection with this survey to a minimum. We give almost no proofs, but provide precise references to these books as well as to the original articles for details, including proofs.

Let me outline the contents of this article. In the first section, we introduce the notion of a distinguished basis of vanishing cycles. More precisely, we define distinguished and weakly distinguished bases. In the second section, we consider the intersection form, the classical monodromy, and the Seifert form and we show how matrices of these invariants with respect to distinguished bases are related to one another. Moreover, we define the concept of Coxeter-Dynkin diagram. In Sect. 3, we consider the change of basis and introduce the action of the braid group on the set of distinguished bases. In Sect. 4, we collect together results about the computation of intersection matrices and Seifert matrices with respect to distinguished bases. In Sect. 5, we discuss the implication of the irreducibility of the discriminant to properties of the 
invariants and we introduce the Lyashko-Looijenga map. In Sect. 6, we review Arnold's classification of singularities and compile explicit results for the simple, unimodal, and bimodal singularities. Sect. 7 is devoted to an algebraic description of the monodromy group. Finally, in Sect. 8, we consider the question to which extent the invariants determine the topological type of the singularities. We conclude with some open problems.

The notion of distinguished bases can also be generalized to isolated complete intersection singularities, see [Eb87]. We shall not discuss this case in this survey, we restrict ourselves to isolated complex hypersurface singularities.

There are many further generalizations and applications of the theory, even outside of singularity theory. We mention some of the results, but mainly indicate references to the corresponding articles. We do not claim to be complete.

Acknowledgements. The author is grateful to C. Hertling for drawing his attention to an error in his book [Eb07] (see the proof of Theorem 8) and for useful discussions.

\section{Distinguished BASES OF VANishing CYCLES}

Let $f:\left(\mathbb{C}^{n+1}, 0\right) \rightarrow(\mathbb{C}, 0)$ be the germ of a holomorphic function with an isolated singularity at the origin. This means that

$$
\operatorname{grad} f(a)=\left(\frac{\partial f}{\partial z_{0}}(a), \ldots, \frac{\partial f}{\partial z_{n}}(a)\right) \neq 0
$$

for all points $a \neq 0$ in a small neighborhood of the origin, $\left(z_{0}, \ldots, z_{n}\right)$ denote the coordinates of $\mathbb{C}^{n+1}$. For short, we call $f$ a singularity.

One has the famous result of Milnor [Mi68a]: Let $\varepsilon>0$ be small enough such that the closed ball $B_{\varepsilon} \subset \mathbb{C}^{n+1}$ of radius $\varepsilon$ around the origin in $\mathbb{C}^{n+1}$ intersects the fiber $f^{-1}(0)$ transversely. Let $0<\eta \ll \varepsilon$ be such that for $t$ in the closed disc $\Delta \subset \mathbb{C}$ of radius $\eta$ around the origin, the fiber $f^{-1}(t)$ intersects the ball $B_{\varepsilon}$ transversely. Let

$$
\begin{aligned}
X_{t} & :=f^{-1}(t) \cap B_{\varepsilon} \text { for } t \in \Delta, \\
X & :=f^{-1}(\Delta) \cap B_{\varepsilon}, \\
X^{*} & :=X \backslash X_{0}, \\
\Delta^{*} & :=\Delta \backslash\{0\} .
\end{aligned}
$$

By a result of J. Milnor [Mi68a], the mapping $\left.f\right|_{X^{*}}: X^{*} \rightarrow \Delta^{*}$ is the projection of a (locally trivial) $\left(C^{\infty}\right)$-differentiable fiber bundle. The fiber $X_{\eta}$ over the point $\eta \in \Delta^{*}$ is a $2 n$-dimensional differentiable manifold with boundary which has the homotopy type of a bouquet of $\mu$ 
$n$-spheres where $\mu$ is the Milnor number of the singularity. This differentiable fiber bundle $\left(X^{*},\left.f\right|_{X^{*}}, \Delta^{*}, X_{\eta}\right)$ is called the Milnor fibration and the typical fiber $X_{\eta}$ is called the Milnor fiber. The only non-trivial reduced homology group is the group $\widetilde{H}_{n}\left(X_{\eta} ; \mathbb{Z}\right)$. It is equipped with the intersection form $\langle$,$\rangle . This bilinear form is symmetric if n$ is even and skew-symmetric if $n$ is odd. We shall only consider homology with integral coefficients and we shall write $\widetilde{H}_{n}\left(X_{\eta}\right)$ for $\widetilde{H}_{n}\left(X_{\eta} ; \mathbb{Z}\right)$ in the sequel.

Definition. The group $\widetilde{H}_{n}\left(X_{\eta}\right)$ together with the intersection form $\langle$,$\rangle is called the Milnor lattice of f$ and denoted by $M$.

The Milnor lattice $M$ is a lattice, i.e., a free $\mathbb{Z}$-module of finite rank equipped with a symmetric or skew-symmetric bilinear form $\langle$,$\rangle . The$ rank of the Milnor lattice is the Milnor number $\mu$.

Let $\omega$ be the loop

$$
\begin{aligned}
\omega:[0,1] & \rightarrow \underset{\mathbb{C}}{ } \\
t & \mapsto \eta e^{2 \pi \sqrt{-1} t} .
\end{aligned}
$$

Then parallel translation along this path induces a diffeomorphism $h=h_{\omega}: X_{\eta} \rightarrow X_{\eta}$ which is called the geometric monodromy of the singularity $f$.

Definition. The induced homomorphism $h_{*}: M \rightarrow M$ on the Milnor lattice $M$ is called the (classical) monodromy (or the (classical) monodromy operator) of the singularity $f$.

Our aim is to study the Milnor fibration, the Milnor lattice $M$, and the monodromy.

For this purpose, we shall consider a morsification of the function $f$. This is defined as follows. An unfolding of $f$ is a holomorphic function germ $F:\left(\mathbb{C}^{n+1} \times \mathbb{C}^{k}, 0\right) \rightarrow(\mathbb{C}, 0)$ with $F(z, 0)=f(z)($ see $[\operatorname{Gr} 19,1.2])$. A morsification is a representative $F: V \times U \rightarrow \mathbb{C}$ of an unfolding

$$
\begin{aligned}
& F:\left(\mathbb{C}^{n+1} \times \mathbb{C}, 0\right) \rightarrow(\mathbb{C}, 0) \\
& (z, \lambda) \quad \mapsto f_{\lambda}(z)
\end{aligned}
$$

of $f$ such that for almost all $\lambda \in U \backslash\{0\}$ (everywhere except from a Lebesgue null set) the function $f_{\lambda}: V \rightarrow \mathbb{C}$ is a Morse function, i.e., has only non-degenerate critical points with distinct critical values. The Morse function $f_{\lambda}$ is itself often called a morsification of $f$. One can show that $f$ has a morsification (see, e.g., [Eb07, Proposition 3.18]).

Let $\lambda$ be chosen so that $f_{\lambda}$ is a Morse function. Let $Y:=f_{\lambda}^{-1}(\Delta) \cap B_{\varepsilon}$ and $Y_{t}:=f_{\lambda}^{-1}(t) \cap B_{\varepsilon}$ for $t \in \Delta$. Assume that $\lambda \neq 0$ is chosen so small that all the critical points are contained in the interior of $Y$ and the fiber 

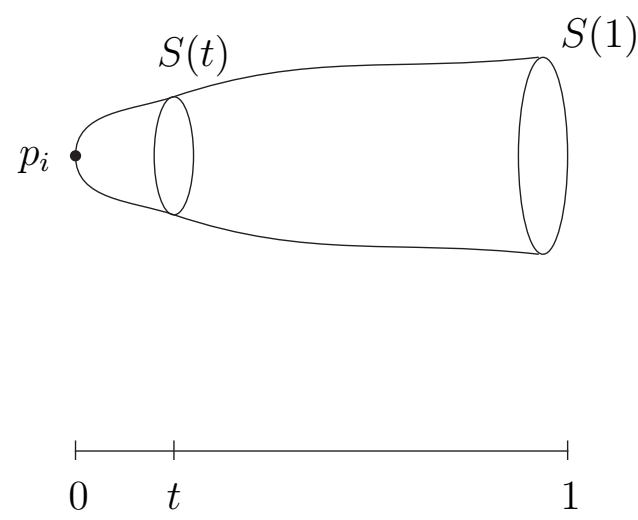

FiguRE 1. Vanishing cycle

$f_{\lambda}^{-1}(t)$ for $t \in \Delta$ intersects the ball $B_{\varepsilon}$ transversely. Denote the critical points by $p_{1}, \ldots, p_{\mu}$ and the critical values by $s_{1}, \ldots, s_{\mu}$. Assume that $\eta \in \partial \Delta$ is a non-critical value of $f_{\lambda}$. Let $\Delta^{\prime}:=\Delta \backslash\left\{s_{1}, \ldots, s_{\mu}\right\}$ and $Y^{\prime}:=$ $Y \cap f_{\lambda}^{-1}\left(\Delta^{\prime}\right)$. Then the mapping $\left.f_{\lambda}\right|_{Y^{\prime}}: Y^{\prime} \rightarrow \Delta^{\prime}$ is the projection of a differentiable fiber bundle. The fiber $Y_{t}$ for $t \in \Delta^{\prime} \cap \Delta^{*}$ is diffeomorphic to $X_{t}$. In particular, $Y_{\eta}$ is diffeomorphic to $X_{\eta}$. We therefore identify these fibers.

Let $\gamma:[0,1] \rightarrow \Delta$ be a piecewise differentiable path which connects the critical value $s_{i}$ with $\eta$ and does not pass through any other critical value, i.e. $\gamma(0)=s_{i}, \gamma(1)=\eta$ and $\gamma((0,1]) \subset \Delta^{\prime}$. By the complex Morse lemma there exists a neighborhood $B_{i}$ of the non-degenerate critical point $p_{i}$ over $s_{i}$ and local coordinates $\left(z_{0}, \ldots, z_{n}\right)$ centered at the point $p_{i}$ such that $f_{\lambda}$ can be written in $B_{i}$ in the form

$$
f_{\lambda}\left(z_{0}, \ldots, z_{n}\right)=s_{i}+z_{0}^{2}+\ldots+z_{n}^{2}
$$

and $B_{i}$ is a ball of radius $\varepsilon$ centered at 0 in these coordinates. For sufficiently small $t>0$ the fiber $X_{\gamma(t)}$ contains an $n$-sphere

$$
S(t):=\sqrt{\gamma(t)-s_{i}} S^{n}
$$

where $S^{n}$ is the $n$-dimensional unit sphere

$$
S^{n}=\left\{\left(z_{0}, \ldots, z_{n}\right) \in \mathbb{C}^{n+1} \mid \operatorname{Im} z_{i}=0, \sum z_{i}^{2}=1\right\} .
$$

By parallel translation along $\gamma$ one obtains an $n$-sphere $S(t) \subset X_{\gamma(t)}$ for each $t \in(0,1]$. For $t=0$ the sphere $S(t)$ shrinks to the critical point $p_{i}$ (cf. Fig. 1). We now choose an orientation of $S(1)$. Then $S(1)$ is an $n$-cycle and represents a homology class $\delta$ in the Milnor lattice $M=\widetilde{H}_{n}\left(X_{\eta}\right)$. 
Definition. The homology class $\delta \in M$ is called a vanishing cycle of $f_{\lambda}$ (along $\gamma$ ). Denote by $\Lambda^{*} \subset M$ the set of vanishing cycles of $f$ (for all possible choices of a morsification, a critical point, a path $\gamma$, and an orientation).

A vanishing cycle is well defined up to orientation.

For the self-intersection number of the vanishing cycle $\delta$ in the Milnor fiber $X_{\eta}$ one has the following result (see also [AGV88, Lemma 1.4], [Eb07, Proposition 5.3]).

Proposition 1. The vanishing cycle $\delta \in M$ has the self-intersection number

$$
\langle\delta, \delta\rangle=(-1)^{n(n-1) / 2}\left(1+(-1)^{n}\right)=\left\{\begin{array}{cl}
0 & \text { for } n \text { odd } \\
2 & \text { for } n \equiv 0(\bmod 4) \\
-2 & \text { for } n \equiv 2(\bmod 4)
\end{array}\right.
$$

Proof. In order to compute the self-intersection number $\langle\delta, \delta\rangle$ of the vanishing cycle $\delta$, it suffices to compute the self-intersection number of the sphere $S^{n}$ in the complex manifold

$$
Z=\left\{\left(z_{0}, \ldots, z_{n}\right) \in \mathbb{C}^{n+1} \mid z_{0}^{2}+\cdots+z_{n}^{2}=1\right\}
$$

It is easy to see that the manifold $Z$ is diffeomorphic to the total space $T S^{n}$ of the tangent bundle of the sphere $S^{n}$ which can be described as follows:

$$
T S^{n}=\left\{u+\sqrt{-1} v \in \mathbb{C}^{n+1} \mid \sum u_{i}^{2}=1, \sum u_{i} v_{i}=0\right\} .
$$

A diffeomorphism from the manifold $Z$ to $T S^{n}$ can be defined by

$$
z_{i}=x_{i}+\sqrt{-1} y_{i} \mapsto u_{i}+\sqrt{-1} v_{i}=\frac{x_{i}}{|x|}+\sqrt{-1} y_{i}
$$

where $|x|=\sqrt{\sum x_{i}^{2}}$. This diffeomorphism sends the unit sphere $S^{n} \subset$ $Z$ to the zero section of the tangent bundle $T S^{n}$. The self-intersection number of the zero section $S^{n}$ in the total space of the tangent bundle $T S^{n}$ is equal to the Euler characteristic $\chi\left(S^{n}\right)=1+(-1)^{n}$. However, the natural orientations of the manifolds $Z$ (as a complex analytic manifold) and $T S^{n}$ (as the total space of a tangent bundle) differ by the sign $(-1)^{n(n-1) / 2}$.

The path $\gamma: I \rightarrow \Delta$ defines a closed path around the critical value $s_{i}$ in the following way: Let $\Delta_{i}$ be a disc of sufficiently small radius $\eta_{i}$ around $s_{i}$ such that $\gamma(I)$ intersects the boundary $\partial \Delta_{i}$ of $\Delta_{i}$ exactly once, namely at time $t=\theta$ at the point $s_{i}+u_{i}$. Let $\tau: I \rightarrow \Delta_{i}$, $t \mapsto s_{i}+u_{i} e^{2 \pi \sqrt{-1} t}$, be the path starting at $s_{i}+u_{i}$ which goes once around $s_{i}$ on the boundary of $\Delta_{i}$ in counterclockwise direction. Moreover, set 


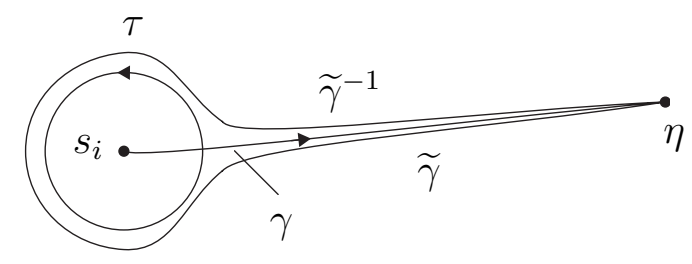

Figure 2. Simple loop associated to $\gamma$

$\widetilde{\gamma}:=\left.\gamma\right|_{[\theta, 1]}$. The closed path $\omega=\widetilde{\gamma}^{-1} \tau \widetilde{\gamma}$ with starting and end point $\eta$ is called the simple loop associated to $\gamma$ (cf. Fig. 2). The monodromy

$$
h_{\delta}:=h_{\omega *}: M \longrightarrow M
$$

corresponding to the simple loop $\omega$ associated to $\gamma$ is called the PicardLefschetz transformation corresponding to the vanishing cycle $\delta$.

The following theorem is the basic result of the Picard-Lefschetz theory. It goes back to Picard and Simart [PS97, p. 95ff.] and Lefschetz [Lef24, Théorème fondamental, p. 23 \& p. 92]. For a proof see [Lam75, §5], [Loo84, Chapter 3], and [AGV88, 1.3]. A proof following the proof in Looijenga's book [Loo84, Chapter 3] is also given in [Eb07, §5.3]. For a modern account of Picard-Lefschetz theory see also the article of Lamotke [Lam81].

Theorem 2 (Picard-Lefschetz formula). For $\alpha \in M$ we have

$$
h_{\delta}(\alpha)=\alpha-(-1)^{\frac{n(n-1)}{2}}\langle\alpha, \delta\rangle \delta .
$$

When $n$ is even, the intersection form $\langle$,$\rangle is a symmetric bilinear$ form and we can combine the formulas from Proposition 1 and Theorem 2 together as

$$
h_{\delta}(\alpha)=\alpha-\frac{2\langle\alpha, \delta\rangle}{\langle\delta, \delta\rangle} \delta
$$

This means that the operator $h_{\delta}: M \rightarrow M$ is a reflection in the hyperplane of $M$ orthogonal to $\delta$. Such a reflection is also denoted by $s_{\delta}$, so in this case $h_{\delta}=s_{\delta}$. When $n$ is odd, the intersection form $\langle$,$\rangle is skew symmetric and Theorem 2$ means that $h_{\delta}$ is a symplectic transvection.

We now assume that $\varepsilon$ and $\eta$ are chosen so small that all the balls $B_{i}$ and all the discs $\Delta_{i}$ are disjoint. We consider an ordered system $\left(\gamma_{1}, \ldots, \gamma_{\mu}\right)$ of paths $\gamma_{i}: I \rightarrow \Delta$ with $\gamma_{i}(0)=s_{i}, \gamma_{i}(1)=\eta$ and $\gamma_{i}((0,1]) \subset \Delta^{\prime}$. 


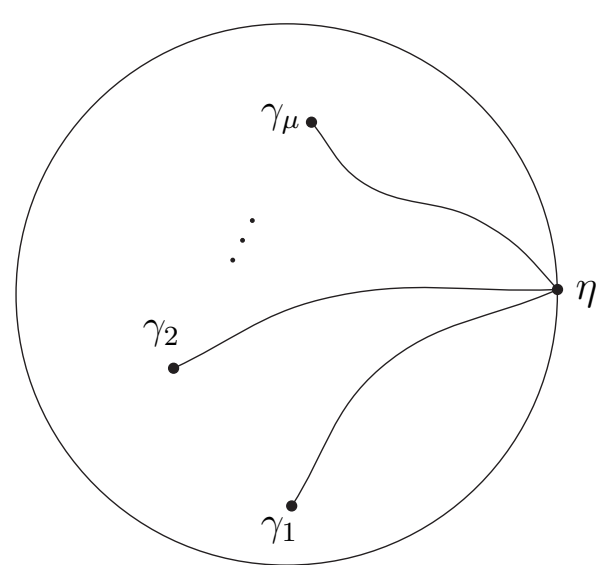

FiguRE 3. Distinguished system of paths

Definition. The system $\left(\gamma_{1}, \ldots, \gamma_{\mu}\right)$ of paths is called distinguished if the following conditions are satisfied:

(i) The paths $\gamma_{i}$ are non-selfintersecting.

(ii) The only common point of $\gamma_{i}$ and $\gamma_{j}$ for $i \neq j$ is $\eta$.

(iii) The paths are numbered in the order in which they arrive at $\eta$ where one has to count clockwise from the boundary of the disc (cf. Fig. 3).

A system $\left(\delta_{1}, \ldots, \delta_{\mu}\right)$ of vanishing cycles $\delta_{i} \in \Lambda^{*}$ is called distinguished, if there exists a distinguished system $\left(\gamma_{1}, \ldots, \gamma_{\mu}\right)$ of paths such that $\delta_{i}$ is a cycle vanishing along $\gamma_{i}$.

Since $\Delta^{\prime}$ is a disc from which $\mu$ points have been deleted, its fundamental group $\pi_{1}\left(\Delta^{\prime}, \eta\right)$ is the free group on $\mu$ generators. If $\left(\gamma_{1}, \ldots, \gamma_{\mu}\right)$ is a distinguished system of paths, then $\pi_{1}\left(\Delta^{\prime}, \eta\right)$ is the free group on the generators $\omega_{1}, \ldots, \omega_{\mu}$, where $\omega_{i}$ is the simple loop associated to $\gamma_{i}$.

Definition. The system $\left(\gamma_{1}, \ldots, \gamma_{\mu}\right)$ of paths is called weakly distinguished if $\pi_{1}\left(\Delta^{\prime}, \eta\right)$ is the free group on the generators $\left(\omega_{1}, \ldots, \omega_{\mu}\right)$, where $\omega_{i}$ is the simple loop belonging to $\gamma_{i}$.

A system $\left(\delta_{1}, \ldots, \delta_{\mu}\right)$ of vanishing cycles $\delta_{i} \in \Lambda^{*}$ is called weakly distinguished if $\delta_{i}$ is a vanishing cycle along a path $\gamma_{i}$ of a weakly distinguished system $\left(\gamma_{1}, \ldots, \gamma_{\mu}\right)$ of paths.

Note that the numbering is important for a distinguished system of paths, but of no significance for a weakly distinguished system of paths. A distinguished system of paths is of course also weakly distinguished.

Brieskorn proved the following theorem [Br70, Appendix] (see also [AGV88, Theorem 2.1], [Eb07, Proposition 5.5]). 
Theorem 3 (Brieskorn). A distinguished system $\left(\delta_{1}, \ldots, \delta_{\mu}\right)$ of vanishing cycles is a basis of the lattice $M$, i.e., $\left\langle\delta_{1}, \ldots, \delta_{\mu}\right\rangle_{\mathbb{Z}}=M$, where $\left\langle\delta_{1}, \ldots, \delta_{\mu}\right\rangle_{\mathbb{Z}}$ denotes the $\mathbb{Z}$-span of $\left(\delta_{1}, \ldots, \delta_{\mu}\right)$.

From this theorem, one can derive the following corollary (see [AGV88, Theorem 2.8], [Eb07, Proposition 5.6]).

Corollary 4. A weakly distinguished system $\left(\delta_{1}, \ldots, \delta_{\mu}\right)$ of vanishing cycles also forms a basis of $M$.

Definition. A basis $\left(\delta_{1}, \ldots, \delta_{\mu}\right)$ of $M$ is called distinguished (resp. weakly distinguished) if $\left(\delta_{1}, \ldots, \delta_{\mu}\right)$ is a distinguished (resp. weakly distinguished) system of vanishing cycles.

By Theorem 3 and Corollary 4 every distinguished or weakly distinguished system of vanishing cycles forms a basis.

The concepts "distinguished" and "weakly distinguished" are due to Gabrielov. In order to distinguish both concepts better, one sometimes says, following a suggestion of Brieskorn, "strongly distinguished" instead of "distinguished". The term "geometric basis" is also used for a distinguished basis.

The group of all automorphisms of a lattice $M$, i.e., isomorphisms $M \rightarrow M$ which respect the bilinear form, will be denoted by $\operatorname{Aut}(M)$.

Definition. The image $\Gamma$ of the homomorphism

$$
\begin{aligned}
\rho: \pi_{1}\left(\Delta^{\prime}, \eta\right) & \longrightarrow \operatorname{Aut}(M) \\
{[\gamma] } & \longmapsto h_{\gamma *}
\end{aligned}
$$

is called the monodromy group of the singularity $f$.

If $\left(\delta_{1}, \ldots, \delta_{\mu}\right)$ is a weakly distinguished basis, then the monodromy group of $f$ is generated by the Picard-Lefschetz transformations $h_{\delta_{i}}$ corresponding to the vanishing cycles $\delta_{i}$. Therefore the monodromy group of $f$ is a group with $\mu$ generators. Indeed, the monodromy group is independent of the morsification of $f$, see Theorem 17 below.

Example 5. (For this example see also [AGV88, 2.9] and [Eb07, Example 5.4].) We consider the function $f: \mathbb{C} \rightarrow \mathbb{C}$ with $f(z)=z^{k+1}$. (This is the singularity $A_{k}$, see Sect. 6.) The Milnor fiber $X_{\eta}$ consists of $k+1$ points, namely the $(k+1)$-th roots of $\eta$. As a morsification of $f$ we consider the function $f_{\lambda}(z)=z^{k+1}-\lambda z$ for $\lambda \neq 0$. Fix $\lambda \in \mathbb{R}$, $\lambda>0$. The critical points of the function $f_{\lambda}$ are given by the equation

$$
f_{\lambda}^{\prime}(z)=(k+1) z^{k}-\lambda=0 .
$$

Therefore they are the points

$$
p_{i}=\sqrt[k]{\frac{\lambda}{k+1}} \xi_{i}, \quad \xi_{i}=e^{-\frac{2 \pi i \sqrt{-1}}{k}},
$$


with the critical values

$$
s_{i}=-\frac{\lambda k}{k+1} \sqrt[k]{\frac{\lambda}{k+1}} \xi_{i}, \quad i=1, \ldots, k
$$

As a noncritical value we choose $-\eta$, where $\eta \in \mathbb{R}, \eta>0$ and

$$
\eta \gg \frac{\lambda k}{k+1} \sqrt[k]{\frac{\lambda}{k+1}} .
$$

Let $\gamma_{i}:[0,1] \rightarrow \bar{\Delta}, t \mapsto(1-t) s_{i}$, and let $\tau$ be a path from 0 to $-\eta$ which runs along the real axis and goes once around the critical value

$$
s_{k}=-\frac{\lambda k}{k+1} \sqrt[k]{\frac{\lambda}{k+1}} \xi_{k} \in \mathbb{R}
$$

in the positive direction.

We consider the path system $\left(\gamma_{1} \tau, \ldots, \gamma_{k} \tau\right)$. This system is homotopic to a distinguished path system. (For the notion of homotopy of path systems see Sect. 3 below.) Let $\left(\delta_{1}, \ldots, \delta_{k}\right)$ be a corresponding distinguished system of vanishing cycles in $\widetilde{H}_{0}\left(X_{-\eta}\right)$.

In order to compute the intersection numbers $\left\langle\delta_{i}, \delta_{j}\right\rangle$ of the vanishing cycles in $\widetilde{H}_{0}\left(X_{-\eta}\right)$ we transport the system $\left(\delta_{1}, \ldots, \delta_{k}\right)$ by parallel transport along the path $\tau^{-1}$ to $\widetilde{H}_{0}\left(X_{0}\right)$. We thus consider a system of vanishing cycles in $\widetilde{H}_{0}\left(X_{0}\right)$, which we again denote by $\left(\delta_{1}, \ldots, \delta_{k}\right)$, and which is defined by the path system $\left(\gamma_{1}, \ldots, \gamma_{k}\right)$.

The fiber $X_{0}$ consists of the $k+1$ points

$$
x_{0}=0, x_{1}=\sqrt[k]{\lambda} \xi_{1}, \ldots, x_{k}=\sqrt[k]{\lambda} \xi_{k} .
$$

Then up to orientation $\delta_{i}$ is represented by the cycle $x_{i}-x_{0}$. It is easy to calculate that $x_{i}-x_{0}$ vanishes along $\gamma_{i}$, i.e., that the points $x_{i}$ and $x_{0}$ fall together along $\gamma_{i}$. Let

$$
\delta_{i}=\left[x_{i}-x_{0}\right], i=1, \ldots, k .
$$

Then

$$
\left\langle\delta_{i}, \delta_{j}\right\rangle= \begin{cases}2 & \text { for } i=j \\ 1 & \text { for } i \neq j .\end{cases}
$$

In this case, the Milnor lattice $M$, the set of vanishing cycles $\Lambda^{*}$, and the monodromy group $\Gamma$ can be described as follows. Let $e_{1}, \ldots, e_{k+1}$ be the standard basis of $\mathbb{R}^{k+1}$ and $\langle$,$\rangle the Euclidean scalar product$ on $\mathbb{R}^{k+1}$. Denote by $\mathcal{S}_{k+1}$ the symmetric group in $k+1$ symbols. Then

$$
\begin{aligned}
M & =\left\{\left(v_{1}, \ldots, v_{k+1}\right) \in \mathbb{Z}^{k+1} \mid v_{1}+\cdots+v_{k+1}=0\right\}, \\
\Lambda^{*} & =\left\{e_{i}-e_{j} \mid 1 \leq i, j \leq k+1, i \neq j\right\}=\{v \in M \mid\langle v, v\rangle=2\}, \\
\Gamma & =\mathcal{S}_{k+1} .
\end{aligned}
$$




\section{Coxeter-Dynkin diagram And Seifert Form}

Definition. Let $\left(\delta_{1}, \ldots, \delta_{\mu}\right)$ be a weakly distinguished basis of $M$. The matrix

$$
S:=\left(\left\langle\delta_{i}, \delta_{j}\right\rangle\right)_{j=1, \ldots, \mu}^{i=1, \ldots, \mu}
$$

is called the intersection matrix of $f$ with respect to $\left(\delta_{1}, \ldots, \delta_{\mu}\right)$.

By Proposition 1, the diagonal entries of the intersection matrix satisfy

$$
\left\langle\delta_{i}, \delta_{i}\right\rangle=(-1)^{\frac{n(n-1)}{2}}\left(1+(-1)^{n}\right) \text { for all } i .
$$

It is usual to represent the intersection matrix by a graph called the Coxeter-Dynkin diagram.

Definition. Let $\left(\delta_{1}, \ldots, \delta_{\mu}\right)$ be a weakly distinguished basis of $M$. The Coxeter-Dynkin diagram of the singularity $f$ with respect to $\left(\delta_{1}, \ldots, \delta_{\mu}\right)$ is the graph $D$ defined as follows:

(i) The vertices of $D$ are in one-to-one correspondence with the elements $\delta_{1}, \ldots, \delta_{\mu}$.

(ii) For $i<j$ with $\left\langle\delta_{i}, \delta_{j}\right\rangle \neq 0$ the $i$-th and the $j$-th vertex are connected by $\left|\left\langle\delta_{i}, \delta_{j}\right\rangle\right|$ edges, weighted with the sign +1 or -1 of $\left\langle\delta_{i}, \delta_{j}\right\rangle \in \mathbb{Z}$. We indicate the weight

$$
w= \begin{cases}(-1)^{\frac{n}{2}} & \text { for } n \text { even, } \\ (-1)^{\frac{n+1}{2}} & \text { for } n \text { odd }\end{cases}
$$

by a dashed line, the weight $-w$ by a solid line.

These diagrams are usually called Dynkin diagrams. However, according to A. J. Coleman [Co89, p. 450], they first appeared in mimeographed notes written by H. S. M. Coxeter (around 1935). Therefore we call them Coxeter-Dynkin diagrams.

Example 6. We continue Example 5. The Coxeter-Dynkin diagram with respect to $\left(\delta_{1}, \ldots, \delta_{k}\right)$ is a complete graph with only dashed edges (i.e., each two vertices are joined by a dashed edge).

If $\left(\delta_{1}, \ldots, \delta_{\mu}\right)$ is a distinguished basis then the classical monodromy operator of $f$ can be expressed as follows:

$$
h_{*}=h_{\delta_{1}} \cdots h_{\delta_{\mu}} .
$$

We call this product the Coxeter element corresponding to the distinguished basis. This follows from the fact that the loop $\omega$ corresponding to $h_{*}$ is homotopic to the combination $\omega_{\mu} \omega_{\mu-1} \cdots \omega_{1}$ of the simple loops associated to $h_{\delta_{\mu}}, h_{\delta_{\mu-1}}, \ldots, h_{\delta_{1}}$.

We have the following algebraic proposition (cf. [Bou02, Ch. V, §6, Exercice 3]). 
Proposition 7. Let $M$ be a free $\mathbb{Z}$-module of rank $\ell$ with a basis $\left(e_{1}, \ldots, e_{\ell}\right)$ and $A=\left(a_{i j}\right)$ an $\ell \times \ell$-matrix with integral coefficients. Consider the operator $s_{i}: M \rightarrow M$ defined by

$$
s_{i}\left(e_{j}\right)=e_{j}-a_{i j} e_{i}
$$

and let $c=s_{1} \cdots s_{\ell}$. Let $C$ be the matrix of $c$ with respect to the basis $\left(e_{1}, \ldots, e_{\ell}\right)$, I the $\ell \times \ell$ unit matrix, and let $U=\left(u_{i j}\right)$ and $V=\left(v_{i j}\right)$ be the matrices defined by

$$
u_{i j}=\left\{\begin{array}{ll}
a_{i j} & \text { if } i<j, \\
0 & \text { otherwise, }
\end{array} \quad v_{i j}= \begin{cases}0 & \text { if } i<j, \\
a_{i j} & \text { otherwise } .\end{cases}\right.
$$

Then

$$
C=(I+U)^{-1}(I-V)
$$

Let $S_{\varepsilon}^{2 n+1}$ be the boundary of the ball $B_{\varepsilon}$. The set $K=f^{-1}(0) \cap S_{\varepsilon}^{2 n+1}$ is called the link of the singularity $f$. Let $T$ be an (open) tubular neighborhood of $K$ in $S_{\varepsilon}^{2 n+1}$. Milnor [Mi68a] has shown that the map

$$
\begin{aligned}
\Phi: S_{\varepsilon}^{2 n+1} \backslash T & \longrightarrow S^{1} \subset \mathbb{C} \\
z & \longmapsto \frac{f(z)}{|f(z)|}
\end{aligned}
$$

is the projection of a differentiable fiber bundle. Moreover, this fibration is equivalent to the restriction of the fibration $\left.f\right|_{X^{*}}: X^{*} \rightarrow \Delta^{*}$ to the boundary $S_{\eta}^{1}$ of $\bar{\Delta}$ [Mi68a, $\left.\S 5\right]$. In particular, the fiber $Z_{w /|w|}:=$ $\Phi^{-1}(w /|w|)$ is diffeomorphic to $X_{w}$ for $w \in S_{\eta}^{1}$. Let $g_{t}: Z_{1} \rightarrow Z_{e^{2 \pi i t}}$ be the parallel transport along $\omega(t)=e^{2 \pi i t}$. For the definition of the linking number see [AGV88, 2.3], [Eb07, 4.7].

Definition. The Seifert form of $f$ is the bilinear form $L$ on $\widetilde{H}_{n}\left(Z_{1}\right) \cong$ $\widetilde{H}_{n}\left(X_{\eta}\right)$ defined by $L(a, b)=l\left(a, g_{1 / 2 *}(b)\right)$ where $l($,$) is the linking$ number.

Let $\left(\delta_{1}, \ldots, \delta_{\mu}\right)$ be a distinguished basis of $f$ and let

- $S:=\left(\left\langle\delta_{i}, \delta_{j}\right\rangle\right)_{j=1, \ldots, \mu}^{i=1, \ldots, \mu}$ be the intersection matrix,

- $L:=\left(L\left(\delta_{i}, \delta_{j}\right)\right)_{j=1, \ldots, \mu}^{i=1, \ldots, \mu}$ be the matrix of the Seifert form, and

- $H$ be the matrix of the monodromy $h_{*}$ with respect to the basis $\left(\delta_{1}, \ldots, \delta_{\mu}\right)$.

Then one has the following theorem.

Theorem 8. The following holds:

(i) The matrix $L$ is a lower triangular matrix with $-(-1)^{n(n-1) / 2}$ on the diagonal.

(ii) $S=-L-(-1)^{n} L^{t}$.

(iii) $H=(-1)^{n+1} L^{-1} L^{t}$. 
Proof. (i) This is [AGV88, Lemma 2.5]. (Note that, according to [AGV88, Remark in 2.5], the matrix of the bilinear form in [AGV88] is written down as the matrix of the corresponding operator and hence corresponds to the transpose matrix in our convention. See also [Eb07, Corollary $5.3(\mathrm{i})$ ], where, unfortunately, there is a misprint: "upper" should be "lower".)

For the proof of (ii) see [AGV88, Theorem 2.4] (see also [Eb07, Corollary 5.3 (ii)]).

(iii) follows from (i) and (ii) by applying Proposition 7.

It follows from Theorem 8 that each of these matrices determines the other two. It is clear that $S$ and $L$ determine the matrix $H$. That the matrix $H$ of the classical monodromy operator with respect to a distinguished basis determines the intersection matrix $S$ was first proved by F. Lazzeri [Laz74] and follows from Theorem 8 and a simple fact in linear algebra [AGV88, Lemma 2.6] (see also [Eb07, Lemma 5.5]).

A. B. Givental [Gi88] introduced $q$-analogues of the invariants and formulas above thus interpolating between the symmetric and skew symmetric versions of these invariants. The $q$-analogue of the monodromy group was studied by G. G. Il'yuta [I196].

\section{Change OF BASIS}

A distinguished or weakly distinguished system $\left(\gamma_{1}, \ldots, \gamma_{\mu}\right)$ of paths can be chosen in many various ways. Next we consider elementary operations on path systems which preserve the property of being distinguished or weakly distinguished.

Let $\left(\gamma_{1}, \ldots, \gamma_{\mu}\right)$ be a distinguished system of paths from the critical values $s_{1}, \ldots, s_{\mu}$ to the non-critical value $\eta$ and let $\left(\delta_{1}, \ldots, \delta_{\mu}\right)$ be a corresponding distinguished system of vanishing cycles. Furthermore, let $\left(\omega_{1}, \ldots, \omega_{\mu}\right)$ be a corresponding system of simple loops.

Definition. The operation $\alpha_{j}$ for $1 \leq j<\mu$ is defined as

$$
\alpha_{j}:\left(\gamma_{1}, \ldots, \gamma_{\mu}\right) \mapsto\left(\gamma_{1}, \ldots, \gamma_{j-1}, \widetilde{\gamma}_{j}, \widetilde{\gamma}_{j+1}, \gamma_{j+2}, \ldots, \gamma_{\mu}\right),
$$

where $\widetilde{\gamma}_{j+1}=\gamma_{j}$ and $\widetilde{\gamma}_{j}$ is a small homotopic deformation of $\gamma_{j+1} \omega_{j}$ such that $\widetilde{\gamma}_{j}$ has no self-intersection points and intersects the other paths only at $\eta$, for $t=1$ (see Fig. 4).

Then $\left(\widetilde{\gamma}_{1}, \ldots, \widetilde{\gamma}_{\mu}\right)$ is again a distinguished system of paths.

This induces the following operation on the corresponding system $\left(\delta_{1}, \ldots, \delta_{\mu}\right)$ of vanishing cycles which will be denoted by the same symbol:

$$
\alpha_{j}:\left(\delta_{1}, \ldots, \delta_{\mu}\right) \mapsto\left(\delta_{1}, \ldots, \delta_{j-1}, h_{\delta_{j}}\left(\delta_{j+1}\right), \delta_{j}, \delta_{j+2}, \ldots, \delta_{\mu}\right)
$$




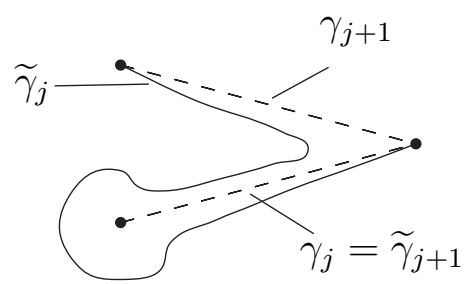

Figure 4. The operation $\alpha_{j}$

where

$$
h_{\delta_{j}}\left(\delta_{j+1}\right)=\delta_{j+1}-(-1)^{n(n-1) / 2}\left\langle\delta_{j+1}, \delta_{j}\right\rangle \delta_{j} .
$$

Definition. The operation $\beta_{j+1}$ for $1 \leq j<\mu$ is defined as

$$
\beta_{j+1}:\left(\gamma_{1}, \ldots, \gamma_{\mu}\right) \mapsto\left(\gamma_{1}, \ldots, \gamma_{j-1}, \gamma_{j}^{\prime}, \gamma_{j+1}^{\prime}, \gamma_{j+2}, \ldots, \gamma_{\mu}\right)
$$

where $\gamma_{j}^{\prime}=\gamma_{j+1}$ and $\gamma_{j+1}^{\prime}$ is a small homotopic deformation of $\gamma_{j} \omega_{j+1}^{-1}$ with the properties above (see Fig. 5). Then $\left(\gamma_{1}^{\prime}, \ldots, \gamma_{\mu}^{\prime}\right)$ is again a

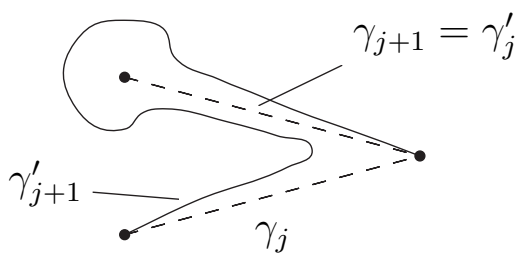

Figure 5. The operation $\beta_{j+1}$

distinguished system of paths.

This induces the following operation on the corresponding system $\left(\delta_{1}, \ldots, \delta_{\mu}\right)$ of vanishing cycles which will also be denoted by the same symbol:

$$
\beta_{j+1}:\left(\delta_{1}, \ldots, \delta_{\mu}\right) \mapsto\left(\delta_{1}, \ldots, \delta_{j-1}, \delta_{j+1}, h_{\delta_{j+1}}^{-1}\left(\delta_{j}\right), \delta_{j+2}, \ldots, \delta_{\mu}\right)
$$

where

$$
h_{\delta_{j+1}}^{-1}\left(\delta_{j}\right)=\delta_{j}-(-1)^{n(n-1) / 2}\left\langle\delta_{j+1}, \delta_{j}\right\rangle \delta_{j+1}
$$

is the inverse Picard-Lefschetz transformation.

Two distinguished systems $\left(\gamma_{1}, \ldots, \gamma_{\mu}\right)$ and $\left(\tau_{1}, \ldots, \tau_{\mu}\right)$ of paths are called homotopic if there are homotopies $\phi_{i}: I \times I \rightarrow \bar{\Delta}$ between $\gamma_{i}$ and $\tau_{i}, i=1, \ldots, \mu$, such that for all $u \in I$ and paths $\phi_{i}^{u}: I \rightarrow \bar{\Delta}$, $t \mapsto \phi_{i}(u, t), i=1, \ldots, \mu$, the following properties are satisfied: 
(i) $\phi_{i}^{u}(0)=s_{i}, \phi_{i}^{u}(1)=\eta$.

(ii) The paths $\phi_{i}^{u}$ are double point free.

(iii) Each two paths $\phi_{i}^{u}$ and $\phi_{j}^{u}$ have, for $i \neq j$, only the end point $\eta$ in common.

One can easily show (see [Eb07, Lemma 6]):

Lemma 9. The operations $\alpha_{j}$ and $\beta_{j+1}$ are mutually inverse, i.e., the application of $\alpha_{j} \beta_{j+1}$ and $\beta_{j+1} \alpha_{j}$ to a distinguished path system $\left(\gamma_{1}, \ldots, \gamma_{\mu}\right)$ yields a homotopic distinguished path system.

Up to homotopy of distinguished path systems we have

(i) $\alpha_{i} \alpha_{j}=\alpha_{j} \alpha_{i}$ for $i, j$ with $|i-j| \geq 2$,

(ii) $\alpha_{j} \alpha_{j+1} \alpha_{j}=\alpha_{j+1} \alpha_{j} \alpha_{j+1}$ for $1 \leq j<\mu-1$.

These are the relations of Artin's braid group [Art25, Art47] (see also [Bir74]). Therefore we have an action of the braid group $\operatorname{Br}_{\mu}$ on $\mu$ strings on the set of the homotopy classes of distinguished path systems and so also on the set of all distinguished systems of vanishing cycles. One can show the following result ([GZ77], see also [Eb07, Proposition 5.15]).

Proposition 10. The braid group $\mathrm{Br}_{\mu}$ acts transitively on the set of all homotopy classes of distinguished path systems, i.e., any two distinguished path systems can be transformed one to the other by iteration of the operations $\alpha_{j}$ and $\beta_{j+1}$ and a succeeding homotopy.

Definition. Let

- $\mathcal{B}$ be the set of all distinguished bases of vanishing cycles of $f$,

- $\mathcal{D}$ be the set of Coxeter-Dynkin diagrams of distinguished bases of $f$.

One also has a braid group action on the sets $\mathcal{B}$ and $\mathcal{D}$. Moreover, one can change the orientation of a cycle. Let $H_{\mu}$ be the direct product of $\mu$ cyclic groups of order two with generators $\kappa_{1}, \ldots, \kappa_{\mu}$, where $\kappa_{i}$ acts on $\mathcal{B}$ by

$$
\kappa_{i}:\left(\delta_{1}, \ldots, \delta_{i}, \ldots, \delta_{\mu}\right) \mapsto\left(\delta_{1}, \ldots,-\delta_{i}, \ldots, \delta_{\mu}\right) .
$$

The braid group $\mathrm{Br}_{\mu}$ acts on $H_{\mu}$ by permutation of the generators $\kappa_{1}, \ldots, \kappa_{\mu}: \alpha_{j}$ corresponds to the transposition of $\kappa_{j}$ and $\kappa_{j+1}$. Let $\mathrm{Br}_{\mu}^{\rtimes}=H_{\mu} \rtimes \mathrm{Br}_{\mu}$ be the semi-direct product. It follows from Proposition 10 that the action of the group $\mathrm{Br}_{\mu}^{\rtimes}$ on $\mathcal{B}$ is transitive.

The set $\mathcal{B}$ depends on the chosen morsification. In order to get an invariant of the singularity, Brieskorn [Br83] proposed a more general notion of distinguished bases. Namely, he considered the natural action of the monodromy group $\Gamma$ on the set $\mathcal{B}$ : An element $h \in \Gamma$ acts as 
follows:

$$
h:\left(\delta_{1}, \ldots, \delta_{\mu}\right) \mapsto\left(h\left(\delta_{1}\right), \ldots, h\left(\delta_{\mu}\right)\right) .
$$

Brieskorn called a basis $B$ of $M$ geometric if it is obtained by any choice of a distinguished path system, of orientations, and of $h \in \Gamma$. He introduced the notions

- $\mathcal{B}^{*}$ for the set of all geometric bases of $f$,

- $\mathcal{D}^{*}$ for the set of Coxeter-Dynkin diagrams of geometric bases of $f$.

The sets $\mathcal{B}^{*}$ and $\mathcal{D}^{*}$ are invariants of the singularity. In fact, the set $\mathcal{D}^{*}$ coincides with $\mathcal{D}$. The action of $\Gamma$ commutes with the action of the group $\mathrm{Br}_{\mu}^{\rtimes}$. It follows from Proposition 10 that the action of the group $\Gamma \times \mathrm{Br}_{\mu}^{\rtimes}$ on $\mathcal{B}^{*}$ is transitive. One can derive from this that the invariants $\mathcal{B}^{*}$ and $\mathcal{D}^{*}$ determine each other, see [Br83].

Note that, unfortunately, in [Eb18] the set $\mathcal{B}$ was considered but denoted by $\mathcal{B}^{*}$.

The braid group action above first appeared in a paper of A. Hurwitz [Hur91] from 1891 where he describes a braid group action on certain sets of Riemann surfaces (cf. [Kl88]). It was also studied by Brieskorn and his students, see [Br88]. In [Br88], Brieskorn introduced a simple unifying concept, the notion of an automorphic set.

Definition. An automorphic set is a set $\Lambda$ with a product $*: \Lambda \times \Lambda \rightarrow$ $\Lambda$ such that all left translations are automorphisms, i.e., one has the following properties:

(i) For all $a, c \in \Lambda$ there is a unique $b \in \Lambda$ such that $a * b=c$.

(ii) For all $a, b, c \in \Lambda$ one has $(a * b) *(a * c)=a *(b * c)$.

The set $\Lambda^{*}$ of vanishing cycles of $f$ is an automorphic set with the product $a * b:=h_{a}(b)$ for $a, b \in \Lambda^{*}$.

If $\Lambda$ is an automorphic set, then one has a canonical braid group action on the $n$-fold cartesian product $\Lambda^{n}$ of $\Lambda$ :

$$
\alpha_{i}:\left(x_{1}, \ldots, x_{n}\right) \mapsto\left(x_{1}, \ldots, x_{i-1}, x_{i} * x_{i+1}, x_{i}, x_{i+2}, \ldots, x_{n}\right) .
$$

The concept of an automorphic set is a basic concept which is also studied under the following names: left self-distributive system, selfdistributive groupoid, quandle, wrack, and rack, see, e.g., the book of P. Dehornoy [Deh00].

This braid group action is also considered in the representation theory of algebras, see, e.g., [CB93, KY11, Rin94]. It has also been applied in mathematical physics, see, e.g., [CV93, FHHI03, FH03].

Example 11. We continue Example 6. By the transformations

$$
\alpha_{k-1}, \alpha_{k-2}, \ldots, \alpha_{1} ; \alpha_{k-1}, \alpha_{k-2}, \ldots, \alpha_{2} ; \ldots ; \alpha_{k-1}, \alpha_{k-2} ; \alpha_{k-1},
$$


the distinguished basis $\left(\delta_{1}, \ldots, \delta_{k}\right)$ is transformed to a distinguished basis with the Coxeter-Dynkin diagram depicted in Fig. 6. This is the classical Coxeter-Dynkin diagram of type $A_{k}$.

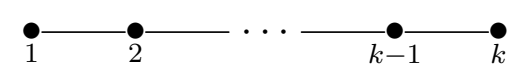

Figure 6. Standard Coxeter-Dynkin diagram $A_{k}$

Finally, we consider operations that transform weakly distinguished path systems again into weakly distinguished path systems.

Let $\left(\gamma_{1}, \ldots, \gamma_{\mu}\right)$ now be a weakly distinguished path system from the points $s_{1}, \ldots, s_{\mu}$ to $\eta$, let $\left(\omega_{1}, \ldots, \omega_{\mu}\right)$ be a corresponding system of simple loops and let $\left(\delta_{1}, \ldots, \delta_{\mu}\right)$ be a corresponding weakly distinguished system of vanishing cycles.

Definition. We define operations $\alpha_{i}(j)$ and $\beta_{i}(j)$ for $i, j \in\{1, \ldots, \mu\}$, $i \neq j$, as follows:

$$
\begin{aligned}
& \alpha_{i}(j):\left(\gamma_{1}, \ldots, \gamma_{\mu}\right) \mapsto\left(\gamma_{1}, \ldots, \gamma_{j-1}, \gamma_{j} \omega_{i}, \gamma_{j+1}, \ldots, \gamma_{\mu}\right) \\
& \beta_{i}(j):\left(\gamma_{1}, \ldots, \gamma_{\mu}\right) \mapsto\left(\gamma_{1}, \ldots, \gamma_{j-1}, \gamma_{j} \omega_{i}^{-1}, \gamma_{j+1}, \ldots, \gamma_{\mu}\right)
\end{aligned}
$$

These operations induce the following operations on the corresponding systems of simple loops, and we denote them by the same symbols:

$$
\begin{aligned}
& \alpha_{i}(j):\left(\omega_{1}, \ldots, \omega_{\mu}\right) \mapsto\left(\omega_{1}, \ldots, \omega_{j-1}, \omega_{i}^{-1} \omega_{j} \omega_{i}, \omega_{j+1}, \ldots, \omega_{\mu}\right), \\
& \beta_{i}(j):\left(\omega_{1}, \ldots, \omega_{\mu}\right) \mapsto\left(\omega_{1}, \ldots, \omega_{j-1}, \omega_{i} \omega_{j} \omega_{i}^{-1}, \omega_{j+1}, \ldots, \omega_{\mu}\right) .
\end{aligned}
$$

If $\omega_{1}, \ldots, \omega_{\mu}$ forms a generating system for $\pi_{1}\left(\Delta^{\prime}, \eta\right)$, then $\pi_{1}\left(\Delta^{\prime}, \eta\right)$ is also generated by the new simple loops that arise from application of the operations $\alpha_{i}(j)$ and $\beta_{i}(j)$. Hence $\alpha_{i}(j)$ and $\beta_{i}(j)$ transfer weakly distinguished path systems again to weakly distinguished path systems.

These operations thus induce operations on the corresponding weakly distinguished systems of vanishing cycles too, which we denote by the same symbols, and they appear as follows:

$$
\begin{aligned}
& \alpha_{i}(j):\left(\delta_{1}, \ldots, \delta_{\mu}\right) \mapsto\left(\delta_{1}, \ldots, \delta_{j-1}, h_{\delta_{i}}\left(\delta_{j}\right), \delta_{j+1}, \ldots, \delta_{\mu}\right), \\
& \beta_{i}(j):\left(\delta_{1}, \ldots, \delta_{\mu}\right) \mapsto\left(\delta_{1}, \ldots, \delta_{j-1}, h_{\delta_{i}}^{-1}\left(\delta_{j}\right), \delta_{j+1}, \ldots, \delta_{\mu}\right) .
\end{aligned}
$$

The operations $\alpha_{i}(j)$ and $\beta_{i}(j)$ are again mutually inverse in the sense above. For even $n$ they even agree. 
If $\left(\gamma_{1}, \ldots, \gamma_{\mu}\right)$ is a distinguished path system and if $\tau_{j, j+1} \in \mathcal{S}_{\mu}$ denotes the transposition of $j$ and $j+1$, then, up to homotopy,

$$
\begin{aligned}
\alpha_{j} & =\tau_{j, j+1} \circ \alpha_{j}(j+1), \\
\beta_{j+1} & =\tau_{j, j+1} \circ \beta_{j+1}(j) .
\end{aligned}
$$

We now also have the following proposition:

Proposition 12. Let $\left(\omega_{1}, \ldots, \omega_{\mu}\right)$ and $\left(\omega_{1}^{\prime}, \ldots, \omega_{\mu}^{\prime}\right)$ be two free generating systems of the free group $\pi_{1}\left(\Delta^{\prime}, \eta\right)$ such that $\omega_{i}$ and $\omega_{i}^{\prime}$ are conjugate to one another for $i=1, \ldots, \mu$. Then one can obtain $\left(\omega_{1}^{\prime}, \ldots, \omega_{\mu}^{\prime}\right)$ from $\left(\omega_{1}, \ldots, \omega_{\mu}\right)$ by the application of a sequence of operations of type $\alpha_{i}(j)$ or $\beta_{i}(j)$.

This proposition was conjectured by Gusein-Zade [GZ77] and proved by S. P. Humphries [Hum85] in 1985. It also follows, as remarked by R. Pellikaan, from an old result of J. H. C. Whitehead from the year 1936 (cf. [LS77, Proposition 4.20]). We refer to [Hum85].

It follows from Proposition 12 that any two weakly distinguished systems of vanishing cycles can be transformed one to the other by iteration of the operations $\alpha_{i}(j)$ and $\beta_{i}(j)$ and a succeeding change of orientation of some of the cycles.

J. McCool [McC86] found a presentation of the subgroup of the automorphism group of a free group generated by the operations $\alpha_{i}(j)$ and $\beta_{i}(j)$.

\section{Computation of intersection matrices}

The Sebastiani-Thom sum of the singularities $f:\left(\mathbb{C}^{n+1}, 0\right) \rightarrow(\mathbb{C}, 0)$ and $g:\left(\mathbb{C}^{m}, 0\right) \rightarrow(\mathbb{C}, 0)$ is the singularity of the function germ $f \oplus g:$ $\left(\mathbb{C}^{n+m+1}, 0\right) \rightarrow(\mathbb{C}, 0)$ defined by the formula

$$
(f \oplus g)(x, y)=f(x)+g(y)
$$

$\left(x \in \mathbb{C}^{n+1}, y \in \mathbb{C}^{m},(x, y) \in \mathbb{C}^{n+m+1} \cong \mathbb{C}^{n+1} \oplus \mathbb{C}^{m}\right)$.

M. Sebastiani and R. Thom [ST71] proved that the monodromy operator of the singularity $f \oplus g$ is equal to the tensor product of the monodromy operators of the singularities $f$ and $g$. If $L_{f}, L_{g}$, and $L_{f \oplus g}$ denote the Seifert form of $f, g$, and $f \oplus g$ respectively, then by a result of Deligne (see [Dem75], see also [AGV88, Theorem 2.10])

$$
L_{f \oplus g}=(-1)^{(n+1) m} L_{f} \otimes L_{g} .
$$

Gabrielov [Ga73] showed how to calculate an intersection matrix of $f \oplus g$ from the intersection matrices of $f$ and $g$ with respect to distinguished 
bases (see also [AGV88, Theorem 2.11]). As a corollary, he obtained certain intersection matrices for singularities of the form

$$
f(x)=z_{0}^{a_{0}}+\cdots+z_{n}^{a_{n}}, \quad \text { for } a_{i} \in \mathbb{Z}, a_{i} \geq 2, i=0, \ldots, n .
$$

These singularities are called Brieskorn-Pham singularities. They were considered by Brieskorn [Br66] and Pham [Ph65] (see also [HM68]). For such a singularity, already Pham [Ph65] had found a basis and calculated the intersection matrix with respect to this basis. Gabrielov showed that Pham's basis can be deformed to a distinguished basis and the intersection matrix is given by the same formulas which Gabrielov obtained. Independently, these intersection matrices with respect to distinguished bases were also calculated by A. Hefez and Lazzeri [HL74].

A special case of the Sebastiani-Thom sum of $f$ and $g$ is the case when $g(y)=y_{1}^{2}+\cdots+y_{m}^{2}$. This is called a stabilization of $f$. The following theorem is a special case of Gabrielov's result (see also [AGV88, Theorem 2.14]).

Theorem 13. Let $f_{\lambda}$ be a morsification of the singularity $f$, let $\left(\gamma_{1}, \ldots, \gamma_{\mu}\right)$ be a distinguished path system for $f_{\lambda}$, and let $\left(\delta_{1}, \ldots, \delta_{\mu}\right)$ be a corresponding distinguished basis.

Then $f_{\lambda}(x)+y_{1}^{2}+\ldots+y_{m}^{2}$ is a morsification of the singularity $f(x)+y_{1}^{2}+\ldots+y_{m}^{2}$, with the same critical values, $\left(\gamma_{1}, \ldots, \gamma_{\mu}\right)$ is also a distinguished path system for this singularity, and for a corresponding distinguished basis $\left(\widetilde{\delta}_{1}, \ldots, \widetilde{\delta}_{\mu}\right)$ we have

$$
\left\langle\widetilde{\delta}_{i}, \widetilde{\delta}_{j}\right\rangle=[\operatorname{sign}(j-i)]^{m}(-1)^{(n+1) m+\frac{m(m-1)}{2}}\left\langle\delta_{i}, \delta_{j}\right\rangle \text { for } i \neq j .
$$

It follows from Theorem 13 that, by taking a suitable stabilization, one can assume that $n \equiv 2 \bmod 4$. In this case, the intersection form is symmetric and the vanishing cycles have self intersection number -2 . The Picard-Lefschetz transformation $h_{\delta_{i}}$ acts on $M$ by the formula

$$
h_{\delta_{i}}(\alpha)=s_{\delta_{i}}(\alpha)=\alpha+\left\langle\alpha, \delta_{i}\right\rangle \delta_{i} .
$$

This is a reflection in the hyperplane orthogonal to the vanishing cycle $\delta_{i}$. In accordance with the definition in Sect. 2, in the Coxeter-Dynkin diagram, edges of weight +1 are depicted by solid lines and edges of weight -1 are depicted by dashed lines. Note that the definition of a Coxeter-Dynkin diagram in $[A G V 88,2.8]$ is slightly different: It encodes the intersection matrix in the case $n \equiv 2 \bmod 4$, and the $i$-th and $j$-th vertices are joined by an edge of multiplicity $\left\langle\delta_{i}, \delta_{j}\right\rangle$.

Example 14. Consider the germ of the function $f: \mathbb{C}^{2} \rightarrow \mathbb{C}$ defined by $f(x, y)=x^{5}+y^{3}$. (This is the singularity $E_{8}$, see Sect. 6.) By 
Example 11 and the result of Gabrielov [Ga73] there is a distinguished basis of $f$ with a Coxeter-Dynkin diagram of the shape of Fig. 7. By

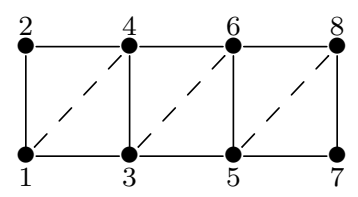

Figure 7. Gabrielov diagram of $E_{8}$

the transformations

$\alpha_{7}, \alpha_{6}, \alpha_{5}, \alpha_{4}, \alpha_{3}, \alpha_{2}, \alpha_{1} ; \beta_{5}, \beta_{4} ; \beta_{7}, \beta_{6}, \beta_{5} ; \beta_{7}, \beta_{6}, \beta_{5} ; \beta_{8}, \beta_{7}, \beta_{6} ; \kappa_{2}, \kappa_{7}, \kappa_{8}$,

the Coxeter-Dynkin diagram is transformed to the classical CoxeterDynkin diagram of type $E_{8}$, see Fig. 8. It follows from Theorem 26 below that the numbering can be changed by braid group transformations to an arbitrary numbering.

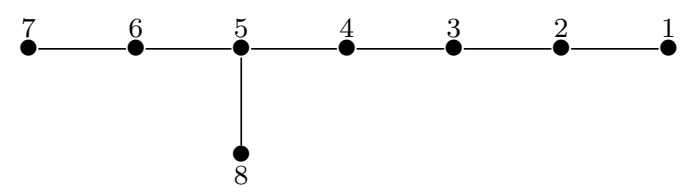

Figure 8. Standard Coxeter-Dynkin diagram $E_{8}$

Another method to compute an intersection matrix with respect to a distinguished basis of $f$ is the polar curve method of Gabrielov [Ga79].

If $n=1$, so $f:\left(\mathbb{C}^{2}, 0\right) \rightarrow(\mathbb{C}, 0)$ defines a curve singularity, there is an especially nice method to compute an intersection matrix with respect to a distinguished basis using a real morsification of the singularity. This method is independently due to N. A'Campo [A'C75a] and Gusein-Zade [GZ74, GZ75].

P. Orlik and R. Randell [OR77] computed the classical monodromy operator for weighted homogeneous polynomials of the form

$$
f\left(z_{0}, \ldots, z_{n}\right)=z_{0}^{a_{0}}+z_{0} z_{1}^{a_{1}}+\ldots+z_{n-1} z_{n}^{a_{n}}, \quad n \geq 1 .
$$

Moreover, they formulated the following conjecture. Let $r_{k}=a_{0} a_{1} \cdots a_{k}$ for $k=0,1, \ldots, n, r_{-1}=1$, and define integers $c_{0}, c_{1}, \ldots, c_{\mu}$ by

$$
\prod_{i=-1}^{n}\left(t^{r_{i}}-1\right)^{(-1)^{n-i}}=c_{\mu} t^{\mu}+\cdots+c_{1} t+c_{0} .
$$


Conjecture 15 (Orlik-Randell). There exists a distinguished basis of $f$ such that the Seifert matrix $L$ of $f$ is given by

$$
L=-(-1)^{n(n+1) / 2}\left(\begin{array}{ccccccc}
c_{0} & 0 & \cdots & \cdots & 0 & 0 & 0 \\
c_{1} & c_{0} & 0 & \cdots & \cdots & 0 & 0 \\
c_{2} & c_{1} & c_{0} & 0 & \cdots & \cdots & 0 \\
\vdots & \vdots & \vdots & \ddots & \ddots & \vdots & \vdots \\
c_{\mu-3} & c_{\mu-4} & c_{\mu-5} & \cdots & c_{0} & 0 & 0 \\
c_{\mu-2} & c_{\mu-3} & c_{\mu-4} & \cdots & c_{1} & c_{0} & 0 \\
c_{\mu-1} & c_{\mu-2} & c_{\mu-3} & \cdots & c_{2} & c_{1} & c_{0}
\end{array}\right)
$$

This conjecture is still open. However, recently D. Aramaki and A. Takahashi [AT19] proved an algebraic analogue of this conjecture.

\section{THE DISCRIMINANT AND THE LyASHKO-LOOIJENGA MAP}

Let $f:\left(\mathbb{C}^{n+1}, 0\right) \rightarrow(\mathbb{C}, 0)$ be a holomorphic function germ with an isolated singularity at $0, \operatorname{grad} f(0)=0$. Then one obtains a universal unfolding $F$ of $f$ as follows (see [Gr19, 1.3] or [Eb07, Proposition 3.17]): Let $g_{0}=-1, g_{1}, \ldots, g_{\mu-1}$ be representatives of a basis of the $\mathbb{C}$-vector space

$$
\mathcal{O}_{n+1} /\left(\frac{\partial f}{\partial z_{0}}, \ldots, \frac{\partial f}{\partial z_{n}}\right) \mathcal{O}_{n+1}
$$

which has dimension $\mu$. Then put

$$
\begin{aligned}
F:\left(\mathbb{C}^{n+1} \times \mathbb{C}^{\mu}, 0\right) & \longrightarrow(\mathbb{C}, 0) \\
(z, u) & \longmapsto f(z)+\sum_{j=0}^{\mu-1} g_{j}(z) u_{j}
\end{aligned}
$$

Let

$$
F: V \times U \rightarrow \mathbb{C}
$$

be a representative of the unfolding $F$, where $V$ is an open neighborhood of 0 in $\mathbb{C}^{n+1}$ and $U$ is an open neighborhood of 0 in $\mathbb{C}^{\mu}$. We put

$$
\begin{aligned}
\mathcal{Y} & :=\{(z, u) \in V \times U \mid F(z, u)=0\}, \\
\mathcal{Y}_{u} & :=\{z \in V \mid F(z, u)=0\} .
\end{aligned}
$$

Since $F(z, 0)=f(z)$, there is an $\varepsilon>0$ such that every sphere $S_{\rho} \subset V$ around 0 of radius $\rho \leq \varepsilon$ intersects the set $\mathcal{Y}_{0}$ transversally. Let $\varepsilon>0$ be so chosen. Then there is also an $\theta>0$ such that for $|u| \leq \theta$ the set $\left\{u \in \mathbb{C}^{\mu}|| u \mid \leq \theta\right\}$ lies entirely in $U$ and $\mathcal{Y}_{u}$ intersects the sphere $S_{\varepsilon}$ 
transversally. Let $\theta$ be so chosen. We put

$$
\begin{aligned}
& \mathcal{X}^{\circ}:=\{(z, u) \in \mathcal{Y}|| z|<\varepsilon,| u \mid<\theta\}, \\
& \mathcal{X}:=\{(z, u) \in \mathcal{Y}|| z|\leq \varepsilon,| u \mid<\theta\}, \\
& \partial \mathcal{X}:=\{(z, u) \in \mathcal{Y}|| z|=\varepsilon,| u \mid<\theta\}, \\
& S:=\{u \in U|| u \mid<\theta\}, \\
& p: \mathcal{X} \longrightarrow S \\
&(z, u) \longmapsto u .
\end{aligned}
$$

Let $C$ be the set the critical points and $D=p(C) \subset S$ the discriminant of $p$. We have the following result (see, e.g., [Eb07, Proposition 3.21]).

Theorem 16. For a suitable $\theta>0$ we have:

(i) The map $p: \mathcal{X} \rightarrow S$ is proper.

(ii) $C$ is a nonsingular analytic subset of $\mathcal{X}^{\circ}$ and is closed in $\mathcal{X}$.

(iii) The restriction $\left.p\right|_{C}: C \rightarrow S$ is finite (i.e., proper with finite fibers).

(iv) The discriminant $D$ is an irreducible hypersurface in $S$.

By the Ehresmann fibration theorem the map

$$
p^{\prime}:=\left.p\right|_{\mathcal{X} \backslash p^{-1}(D)}: \mathcal{X} \backslash p^{-1}(D) \longrightarrow S \backslash D
$$

is then the projection of a differentiable fiber bundle, and the fibers of $p^{\prime}$ are diffeomorphic to a Milnor fiber $X_{\eta}$ of $f$.

Let $s \in S \backslash D$ and $X_{s}:=p^{-1}(s)=\left(p^{\prime}\right)^{-1}(s)$. Then $p^{\prime}$ defines a representation

$$
\rho: \pi_{1}(S \backslash D, s) \longrightarrow \operatorname{Aut}\left(\widetilde{H}_{n}\left(X_{s}\right)\right) .
$$

One has the following theorem (see [AGV88, Theorem 3.1] or [Eb07, Proposition 5.17]).

Theorem 17. The image $\Gamma$ of the homomorphism

$$
\rho: \pi_{1}(S \backslash D, s) \longrightarrow \operatorname{Aut}\left(\widetilde{H}_{n}\left(X_{s}\right)\right)
$$

coincides with the monodromy group of the singularity.

In particular, Theorem 17 implies that the monodromy group is independent of the chosen morsification.

As a corollary of the irreducibility of the discriminant (Theorem 16(iv)) and Theorem 17 we obtain the following result which was first proved by Gabrielov [Ga74a] and independently by Lazzeri [Laz73, Laz74]. 
Corollary 18 (Gabrielov, Lazzeri). The Coxeter-Dynkin diagram with respect to a weakly distinguished system $\left(\delta_{1}, \ldots, \delta_{\mu}\right)$ of vanishing cycles is a connected graph.

One can show that if 0 is neither a regular nor a non-degenerate critical point of $f$, then there are two vanishing cycles $\delta, \delta^{\prime}$ of $f$ with $\left\langle\delta, \delta^{\prime}\right\rangle=1$. This follows from the following result due to G. N. Tyurina [Ty68, Theorem 1] and D. Siersma [Si74, Proposition (8.9)] (see also [AGV88, Theorem 3.23], [Eb07, 5.9]) and the fact that, if 0 is neither a regular nor a non-degenerate critical point of $f$, then $f$ deforms to the singularity $g$ with $g(z)=z_{0}^{3}+z_{1}^{2}+\cdots+z_{n}^{2}$.

Theorem 19 (Tyurina, Siersma). Let $f_{t}:\left(\mathbb{C}^{n+1}, 0\right) \rightarrow(\mathbb{C}, 0), t \in$ $[0,1]$, be a continuous deformation of the singularity $f_{0}=f$ with $\mu\left(f_{0}\right)=\mu, \mu\left(f_{t}\right)=\mu^{\prime}$ for $0<t \leq 1$. Then one has $\mu \geq \mu^{\prime}$, one has a natural inclusion of the Milnor lattice $M_{f_{t}}$ of $f_{t}$ in the Milnor lattice $M_{f_{0}}$ of $f_{0}$, and a distinguished basis of $f_{t}$ can be extended to a distinguished basis of $f_{0}$.

From these results one obtains another corollary of the irreducibility of the discriminant (cf. [AGV88, Theorem 3.4], [Eb07, Proposition 5.20]):

Corollary 20. If not both (i) $n$ is odd and (ii) 0 is a non-degenerate critical point of $f$, then the set of vanishing cycles $\Lambda^{*}$ is the only $\Gamma$-orbit, i.e., the monodromy group $\Gamma$ acts transitively on $\Lambda^{*}$.

Using these results, K. Saito [Sa82] showed that the monodromy group $\Gamma$ determines the Milnor lattice $M$.

We can also deduce from these results that the classical monodromy operator acts irreducibly (cf. [AGV88, Theorem 3.5]). (An earlier result for curves was obtained by C. H. Bey [Bey72a, Bey72b].)

Corollary 21. Let $\left(\delta_{1}, \ldots, \delta_{\mu}\right)$ be a distinguished basis of $f$ and let $I$ be a subset of the set of indices $I_{0}=\{1, \ldots, \mu\}$ such that the linear span of the basis elements $\delta_{i}$ with $i \in I$ is invariant under the classical monodromy operator $h_{*}$. Then either $I=\emptyset$ or $I=I_{0}$.

Corollary 22. If the classical monodromy operator of a singularity is the multiplication by \pm 1 , then the singularity is non-degenerate.

This was first proved by A'Campo [A'C73, Théorème 2] as an answer to a question of Sebastiani. It was deduced from the following result.

Theorem 23 (A'Campo). The trace of the classical monodromy operator of $f$ is

$$
\operatorname{tr} h_{*}=(-1)^{n}
$$


The corank of a singularity $f$ is the corank of the Hesse matrix of $f$. Using a result of Deligne (see $\left[\mathrm{A}^{\prime} \mathrm{C} 75 \mathrm{c}\right]$ ), the author proved the following result [Eb96, Proposition 5].

Proposition 24. Let $n \equiv 2 \bmod 4$ and let $c(f)$ denote the corank of $f$. Then

$$
\operatorname{tr} h_{*}^{2}=(-1)^{c(f)} .
$$

A very important result on the classical monodromy is the following theorem.

Theorem 25 (Monodromy theorem). The classical monodromy of $f$ is quasi-unipotent, i.e., its eigenvalues are roots of unity.

For the history of this theorem and further properties of the classical monodromy see the survey article [Eb06]. The usual proofs of Theorem 25 use a resolution of the singularity, see e.g. [EG07] for an instructive one. For a proof which does not use a resolution see [Le78].

The bifurcation variety Bif is the set of all $\lambda \in S$ such that $f_{\lambda}$ does not have $\mu$ distinct critical values. Looijenga [Loo74] in 1974 and independently Lyashko (in the same year, but his work was only published later in [Ly79, Ly84]) introduced the following mapping: The Lyashko-Looijenga mapping LL sends a point $\lambda \in S$ to the unordered collection of critical values of the function $f_{\lambda}$ or, what amounts to the same thing but is sometimes more convenient, to the polynomial which has these critical values as roots. If $\mathcal{P}^{\mu}$ denotes the set of monic polynomials of degree $\mu$, then this is the mapping

$$
\begin{aligned}
\mathrm{LL}: S & \longrightarrow \mathcal{P}^{\mu} \\
\lambda & \longmapsto \prod_{i=1}^{\mu}\left(t-s_{i}\right)
\end{aligned}
$$

where $s_{1}, \ldots, s_{\mu}$ are the critical values of the function $f_{\lambda}$. Let $\Sigma \subset \mathcal{P}^{\mu}$ denote the discriminant variety in $\mathcal{P}^{\mu}$. Then there exists a neighborhood $U \subset S$ of $0 \in S$ such that LL $\left.\right|_{U \backslash \text { Bif }}: U \backslash$ Bif $\rightarrow \mathcal{P}^{\mu} \backslash \Sigma$ is locally biholomorphic [Loo74, Theorem (1.4)].

\section{Special Singularities}

We shall now consider what is known about these invariants for special classes of singularities.

Let $f, g:\left(\mathbb{C}^{n+1}, 0\right) \rightarrow(\mathbb{C}, 0)$ be holomorphic function germs with an isolated singularity at 0 . The germs $f$ and $g$ are called right equivalent if $f$ is taken to $g$ under (the germ of) a biholomorphic mapping of the domain space which leaves the origin invariant. The modality (or module number) of $f$ is the smallest number $m$ for which there exists a representative $p: \mathcal{X} \rightarrow S$ of the universal unfolding $F:\left(\mathbb{C}^{n+1} \times \mathbb{C}^{\mu}, 0\right) \rightarrow(\mathbb{C}, 0)$ 


\begin{tabular}{lllll}
\hline$A_{k}: x^{k+1}, k \geq 1$ & $D_{k}: x^{2} y+y^{k-1}, k \geq 4$ & \\
$E_{6}: x^{3}+y^{4}$ & $E_{7}: x^{3}+x y^{3}$ & $E_{8}: x^{3}+y^{5}$ \\
\hline
\end{tabular}

TABLE 1. Simple singularities

of $f$ such that for all $(z, u) \in \mathcal{X}$ the function germs $F_{u}:\left(\mathbb{C}^{n+1}, z\right) \rightarrow$ $(\mathbb{C}, F(z, u))$ given by $F_{u}\left(z^{\prime}\right)=F\left(z^{\prime}, u\right)$ fall into finitely many families of right equivalence classes depending on at most $m$ (complex) parameters. Singularities of modality 0,1 and 2 are called simple, unimodal (or unimodular), and bimodal (or bimodular), respectively.

V. I. Arnold classified the singularities up to modality 2 [Arn75]. He listed certain normal forms. A normal form determines a class of singularities. This class corresponds to a $\mu=$ const stratum: Any two singularities of a $\mu=$ const stratum are $\mu$-equivalent, see Sect. 8 below. By Proposition 40 below, the class $\mathcal{D}$ is the same for all singularities of a $\mu=$ const stratum. Gabrielov [Ga74a] proved that the dimension of the $\mu=$ const stratum is equal to the modality of the singularity. Arnold found that in the lists of classes, all the classes are split into series which are now called the Arnold series. However, as Arnold writes in [Arn75], "although the series undoubtedly exist, it is not at all clear what a series is". Let us look at Arnold's classification.

Let us first assume that $f:\left(\mathbb{C}^{n+1}, 0\right) \rightarrow(\mathbb{C}, 0)$ defines a simple singularity. Up to stabilization, the simple singuarities are given by the germs of the functions of Table 1 . There are many characterizations of simple singularities, see [Du79]. They are the only singularities where, for $n \equiv 2 \bmod 4$, the intersection form is negative definite [Du79, Characterization B5]. They are also the only singularities where the set $\mathcal{D}$ contains a tree [Du79, Characterization B7], [A'C75b, A'C76]. Moreover, this is also the only case where the monodromy group $\Gamma$ is finite [Du79, Characterization B8]. The author [Eb18] has recently shown that they are the only singularities where the set $\mathcal{B}$ is finite.

Let $f:\left(\mathbb{C}^{n+1}, 0\right) \rightarrow(\mathbb{C}, 0)$ define a simple singularity and $n \equiv$ $2 \bmod 4$. Then $\Lambda^{*}$ is a root system of type $A_{k}, D_{k}, E_{k}$. (Note that the usual bilinear form of [Bou02] has to be multiplied by -1 .) The Milnor lattice $M$ is the corresponding root lattice, the group $\Gamma$ is the corresponding Weyl group, and the classical monodromy operator $h_{*}$ is a Coxeter element of the corresponding root system. Let $c \in \Gamma$ be a Coxeter element. Define

$$
\Xi_{c}:=\left\{\left(s_{1}, \ldots, s_{k}\right) \mid s_{i} \in \Gamma \text { reflection, } s_{1} \cdots s_{k}=c\right\} .
$$

Deligne [Del74] in a letter to Looijenga (with the help of J. Tits and D. Zagier) showed the following theorem. 
Theorem 26 (Deligne). The braid group $\mathrm{Br}_{k}$ acts transitively on $\Xi_{c}$.

From this we obtain the following result.

Corollary 27. One has

$$
\mathcal{B}=\left\{\left(\delta_{1}, \ldots, \delta_{k}\right) \in\left(\Lambda^{*}\right)^{k} \mid\left\langle\delta_{1}, \ldots, \delta_{k}\right\rangle_{\mathbb{Z}}=M, s_{\delta_{1}} \cdots s_{\delta_{k}}=h_{*}\right\} .
$$

The sets $\Xi_{c}, \mathcal{B}$, and $\mathcal{D}$ are finite sets. The cardinality of these sets was calculated in the letter of Deligne (see also [Vo85b, Kl89]).

Example 28. For $E_{8}$ one has $\left|\mathcal{D}_{E_{8}}\right|=2^{8} 3^{4} 5^{6}=324000000$.

The first published proof of Theorem 26 is due to D. Bessis and can be found in [Bes03]. This theorem has been generalized and it has also applications outside of singularity theory, see [BDSW14]. K. Igusa and R. Schiffler generalized this result to arbitrary Coxeter groups of finite rank [IS10, Theorem 1.4] (see also [BDSW14, BGRW17]). Recently, B. Baumeister, P. Wegener, and S. Yahiatene [BWY19] generalized it to certain extended Weyl groups (see below). The theorem has applications in the theory of Artin groups, see [Bes03, Di06], and in the representation theory of algebras, see [IS10, Ig11, HK16].

Let $\mathbb{R}^{k}$ be a vector space on which the Weyl group $W=\Gamma$ acts in a canonical way and let $\mathbb{C}^{k}=\mathbb{R}^{k} \otimes_{\mathbb{R}} \mathbb{C}$ be its complexification. The action of $W$ on $\mathbb{R}^{k}$ extends in a natural way to an action of $W$ on $\mathbb{C}^{k}$. Let $H$ be be union of the complexifications of the reflection hyperplanes of $W$. Let $S$ be the base space and $D$ the discriminant of the universal unfolding of the simple singularity $f$. Then the pair $(S, D)$ is analytically isomorphic (in a neighborhood of the origin) to the pair $\left(\mathbb{C}^{k} / W, H / W\right)[$ Arn72]. Brieskorn [Br71] proved that the fundamental group of the space $\mathbb{C}^{k} / W \backslash H / W$ is the generalized Brieskorn braid group $\pi$ [Br73a, BS72] of the Weyl group $W$. He conjectured [Br73a] and Deligne [Del72] proved that this space is in fact a $K(\pi, 1)$-space. (A $K(\pi, 1)$-space is a topological space with fundamental group $\pi$ and trivial higher homotopy groups.) From this it follows that the complement $S \backslash D$ is a $K(\pi, 1)$-space as well (see also [AGV88, Theorem 3.9]). Brieskorn asked [Br73b, Problème 15] whether this is true in general.

Now we consider the Lyashko-Looijenga map in the case of the simple singularities. Looijenga [Loo74] and Lyashko [Ly79, Ly84] showed that the mapping LL $\left.\right|_{U \backslash \text { Bif }}: U \backslash$ Bif $\rightarrow \mathcal{P}^{k} \backslash \Sigma$ is a covering of degree

$$
d=\frac{k ! N^{k}}{|\Gamma|}
$$

where $N$ is the Coxeter number of the corresponding root system. I. S. Livshits [Li81] determined the Galois group of this covering (see 


\begin{tabular}{lll}
\hline$\widetilde{E}_{6}:$ & $x^{3}+y^{3}+z^{3}+a x y z$, & $a^{3}+27 \neq 0$ \\
$\widetilde{E}_{7}:$ & $x^{2}+y^{4}+z^{4}+a y^{2} z^{2}$, & $a^{2} \neq 4$ \\
$\widetilde{E}_{8}:$ & $x^{2}+y^{3}+z^{6}+a y^{2} z^{2}$, & $4 a^{3}+27 \neq 0$ \\
\hline
\end{tabular}

TABLE 2. Simple elliptic singularities

also [Yu99]). Let $p \in \mathcal{P}^{k} \backslash \Sigma$. It is well known that

$$
\pi_{1}\left(\mathcal{P}^{k} \backslash \Sigma, p\right) \cong \mathrm{Br}_{k}
$$

Therefore the complement of the bifurcation variety of a simple singularity is a $K(\pi, 1)$, where $\pi$ is a subgroup of index $d$ in the braid group $\mathrm{Br}_{k}$.

Similar questions were also answered for complex reflection groups, see [Bes15, Rip10, Rip12].

Looijenga already proved Theorem 26 in the case $A_{k}$ [Loo74, Corollary (3.8)]. Moreover, in this case, he established a correspondence between generic polynomial coverings of the complex sphere and trees with totally ordered edges. By considering a generalized version of the Lyashko-Looijenga mapping, more general combinatorial results were obtained by Arnold [Arn96], D. Zvonkine and S. K. Lando [ZL99], and B. S. Bychkov [By15].

By studying the Lyashko-Looijenga mapping, Jianming Yu [Yu96] determined the number of Seifert matrices with respect to distinguished bases of a simple singularity.

Gusein-Zade [GZ80] gave a characterization of distinguished bases for simple singularities. Let $f:\left(\mathbb{C}^{n+1}, 0\right) \rightarrow(\mathbb{C}, 0)$ define a simple singularity of Milnor number $\mu$. He showed that, if $\left(\delta_{1}, \ldots, \delta_{\mu}\right)$ is an integral basis of the homology group $M$ in which the matrix of the Seifert form is lower triangular, then $\left(\delta_{1}, \ldots, \delta_{\mu}\right)$ is a distinguished basis of vanishing cycles. The proof is based on the following result: Let $n$ be even. For any vanishing cycle $\delta$ and any distinguished basis $\left(\delta_{1}, \ldots, \delta_{\mu}\right)$ for $f$, there exists a distinguished basis $\left(\delta_{1}^{\prime}, \ldots, \delta_{\mu}^{\prime}\right)$ with the first element $\delta_{1}^{\prime}= \pm \delta$. H. Serizawa [Se01] showed that the latter result is false for a non-simple singularity.

The next case are the simple elliptic singularities (see also [Sa74]). These are the singularities $\widetilde{E}_{6}, \widetilde{E}_{7}$, and $\widetilde{E}_{8}$. Up to stabilization, they are given by the one-parameter families of Table 2. These singularities can be characterized as follows: For $n \equiv 2 \bmod 4$, the intersection form is not negative definite but negative semi-definite [Du79, Characterization C5]. Therefore, the simple elliptic singularities are also called the parabolic singularities. The monodromy group is not finite 
but has polynomial growth [Du79, Characterization C6]. (For the notions of polynomial and exponential growth, see e.g. [Mi68b]. See also Remark 35 below.) The set $\mathcal{B}$ is infinite but the set $\mathcal{D}$ is finite [Eb18]. Let $f:\left(\mathbb{C}^{n+1}, 0\right) \rightarrow(\mathbb{C}, 0)$ define a simple elliptic singularity of type $\widetilde{E}_{k}, k=6,7,8$, and let $n \equiv 2 \bmod 4$. If $M$ is a lattice, denote by $M^{\#}=\operatorname{Hom}(M, \mathbb{Z})$ the dual module and by

$$
\begin{aligned}
j: M & \longrightarrow M^{\#} \\
v & \longmapsto l_{v} \text { with } l_{v}(x)=\langle v, x\rangle, x \in M,
\end{aligned}
$$

the canonical homomorphism. The Milnor lattice $M$ is the orthogonal direct sum of the root lattice $E_{k}$ and the radical ker $j$ which is twodimensional. The set $\Lambda^{*}$ of vanishing cycles is an extended affine root system of type $E_{k}^{(1,1)}$ in the sense of Saito [Sa85]. The monodromy group $\Gamma$ is the semi-direct product of the group ker $j \otimes j(M)$ and the Weyl group $W\left(E_{k}\right)$ of $E_{k}$, where the group ker $j \otimes j(M)$ acts on $M$ by $\left(v \otimes w^{\#}\right)(x)=x+w^{\#}(x) v$ and the action of $W\left(E_{k}\right)$ on $\operatorname{ker} j \otimes j(M)$ is trivial on the first factor and canonical on the second one, see [Loo78, Proposition (6.7)]. It follows from this description that the monodromy group has polynomial growth.

P. Kluitmann extended Corollary 27 to the simple elliptic singularities $[K 186]$. He also calculated the cardinality of $\mathcal{D}$ for $\widetilde{E}_{6}$ and $\widetilde{E}_{7}$. In [Jaw86, Jaw88], P. Jaworski considered the Lyashko-Looijenga map for the simple elliptic singularities and showed that the complement of the bifurcation variety of a simple elliptic singularity is again a $K(\pi, 1)$ for a certain subgroup of the braid group $\mathrm{Br}_{\mu}$ [Jaw86, Corollary 2]. Recently, C. Hertling and C. Roucairol [HR18] used a different approach to study the Lyashko-Looijenga map for the simple and simple elliptic singularities and refined and extended the results of Kluitmann and Jaworski.

For the remaining singularities, the sets $\mathcal{B}$ and $\mathcal{D}$ are infinite [Eb18]. Let $f:\left(\mathbb{C}^{n+1}, 0\right) \rightarrow(\mathbb{C}, 0)$ be such a singularity. We assume $n \equiv$ $2 \bmod 4$. The only singularities with a hyperbolic intersection form, i.e., an indefinite form with only one positive eigenvalue, are the singularities of the series $T_{p, q, r}$ with $2 \leq p \leq q \leq r$ and $\frac{1}{p}+\frac{1}{q}+\frac{1}{r}<1$ [Arn73]. Up to stable equivalence, they are given by the one parameter families

$$
T_{p, q, r}: x^{p}+y^{q}+z^{r}+a x y z, a \neq 0 .
$$

They are also called the hyperbolic singularities. The simple elliptic and hyperbolic singularities are unimodal singularities. Gabrielov [Ga74b] calculated Coxeter-Dynkin diagrams with respect to distinguished bases for the unimodal singularities. According to [Ga74b], 


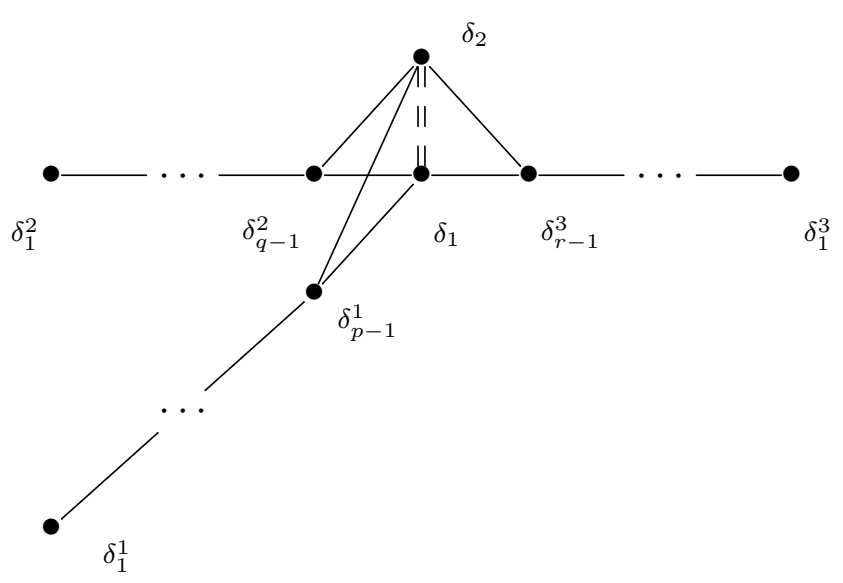

Figure 9. The graph $T_{p, q, r}$

the simple elliptic and hyperbolic singularities have Coxeter-Dynkin diagrams with respect to distinguished bases in the form of Fig. 9. Here $(p, q, r)=(3,3,3),(2,4,4),(2,3,6)$ for $\widetilde{E}_{6}, \widetilde{E}_{7}$, and $\widetilde{E}_{8}$ respectively. The Milnor lattice $M$ of a hyperbolic singularity has a one-dimensional radical ker $j$ generated by the vector $\delta_{2}-\delta_{1}$. By [Ga74b], the monodromy group $\Gamma$ is the semi-direct product of the group $\operatorname{ker} j \otimes j(M)$ and the Coxeter group corresponding to the graph of Fig. 9 with the vertex $\delta_{2}$ removed. It can also be described as the extended Weyl group of a generalized root system as defined by Looijenga [Loo80].

As an application of [BWY19], one obtains an extension of Corollary 27 to the hyperbolic singularities.

Looijenga ([Loo80], [Loo81, Chapter III.3]) gave a description of the complement of the discriminant of a simple elliptic or hyperbolic singularity as an orbit space $Y / \Gamma$. Using this, H. van der Lek [vdL83] gave a presentation of the fundamental group of the discriminant complement for such singularities generalizing the results for the simple singularities.

Besides these singularities, there are 14 exceptional unimodal singularities. Equations of these singularities can be found in $[$ Arn75, AGV85]. Coxeter-Dynkin diagrams for these singularities were also calculated by Gabrielov [Ga74b]. He claimed that by change-of-basis transformations $\alpha_{i}(j)$ and $\beta_{i}(j)$, the Coxeter-Dynkin diagrams can be reduced to the "normal form" depicted in Fig. 10 for certain triples $(p, q, r)$. The author showed in his PhD thesis [Eb80] that these di- 


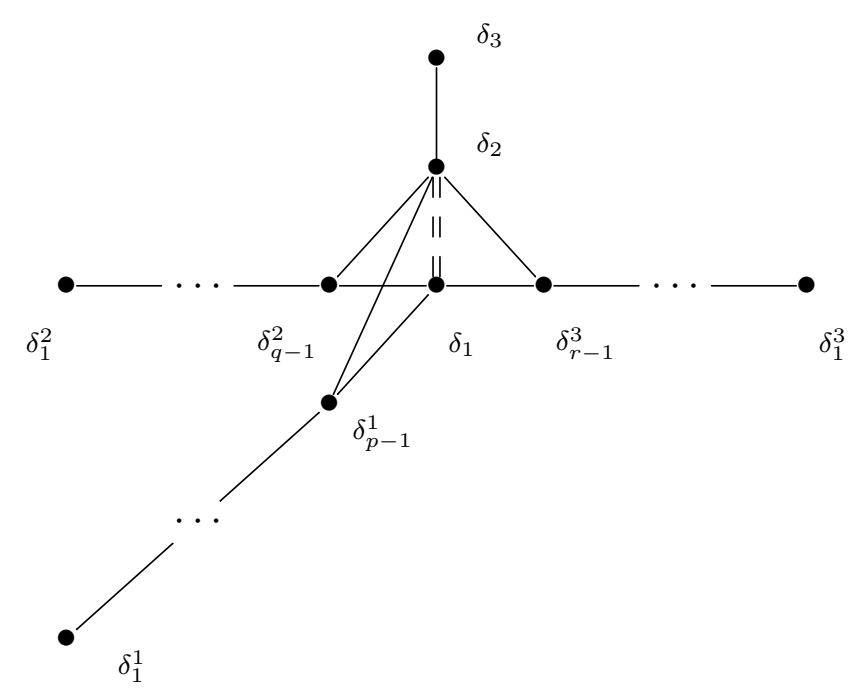

FiguRE 10. The graph $S_{p, q, r}$

agrams are in fact Coxeter-Dynkin diagrams with respect to distinguished bases. The necessary braid group transformations are indicated in the appendix of the thesis which is not published in [Eb81]. However, they can be found in the paper [Eb96]. Arnold observed a strange duality between the 14 exceptional unimodal singularities [Arn75]. The relation to homological mirror symmetry is explained in the survey article [Eb17]. For a description of the monodromy groups see Sect. 7.

V. I. Arnold also classified the bimodal singularities [Arn75, AGV85]. Gabrielov computed Coxeter-Dynkin diagrams with respect to distinguished bases for the singularities of all the series of Arnold, including the bimodal singularities [Ga79]. The author suggested a "normal form" for the Coxeter-Dynkin diagrams with respect to distinguished bases for the bimodal singularities [Eb81, Eb83b]. Jointly with D. Ploog [EP13], he gave a geometric construction of these diagrams. Moreover, he suggested a "normal form" for the Coxeter-Dynkin diagrams with respect to weakly distinguished bases of all the singularities of Arnold's series and calculated their intersection forms [Eb81].

Il'yuta [Il87] formulated two conjectures relating the shape of Coxeter-Dynkin diagrams to the modality of the singularity. He used the definition of the Coxeter-Dynkin diagram of [AGV88, 2.8]: It is a graph with simple edges where the edge between $\delta_{i}$ and $\delta_{j}$ has the weight $\left\langle\delta_{i}, \delta_{j}\right\rangle$. (We assume $n \equiv 2 \bmod 4$.) A monotone cycle in a CoxeterDynkin diagram is a sequence of vertices $\left(\delta_{i_{1}}, \ldots, \delta_{i_{k}}\right)$ where $i_{1}<i_{2}<$ 
$\ldots<i_{k}$ and $\delta_{i_{j}}$ is connected to $\delta_{i_{j+1}}$ for $j=1, \ldots, k$ and $j+1$ taken modulo $k$. The weight of a monotone cycle is the product $\prod\left\langle\delta_{i_{j}}, \delta_{i_{j+1}}\right\rangle$ where the product is over $j=1, \ldots, k$ and $j+1$ taken modulo $k$. Now Il'yuta conjectured:

Conjecture 29 (Il'yuta). The minimum over all $D \in \mathcal{D}$ of the smallest number of edges that have to be deleted in order that $D$ does not contain monotone cycles is equal to the modality of the singularity.

Conjecture 30 (Il'yuta). The minimum over all $D \in \mathcal{D}$ of the number of edges of $D$ of negative weight is equal to the modality of the singularity.

Il'yuta showed that these conjectures hold for the unimodal singularities. The author [Eb96] showed that both conjectures are even true for the unimodal singularities if one counts an edge of weight $\left\langle\delta_{i}, \delta_{j}\right\rangle$ as $\left|\left\langle\delta_{i}, \delta_{j}\right\rangle\right|$ edges as in the definition of the Coxeter-Dynkin diagram in Sect. 2. However, he gave counterexamples to both conjectures for the bimodal singularities. Il'yuta also found other characterizations of Coxeter-Dynkin diagrams of the simple singularities [I194, I195, I197].

Using the Lyashko-Looijenga mapping, M. Lönne [Loe07, Loe10] gave a presentation of the fundamental group of the discriminant complement for Brieskorn-Pham singularities which is related to the intersection matrix with respect to a distinguished basis considered in Sect. 4.

V. A. Vassiliev listed some problems about the Lyashko-Looijenga mapping for non-simple singularities in [Va15].

\section{The MONODROMY GROUP}

In this section we give a description of the monodromy group in the general case.

Let $M$ be a lattice which is either symmetric and even or skew symmetric. Let $\varepsilon \in\{+1,-1\}$ and let $\Lambda$ be a subset of $M$. If $M$ is symmetric we demand that $\langle\delta, \delta\rangle=2 \varepsilon$ for all $\delta \in \Lambda$. We define an automorphism $s_{\delta} \in \operatorname{Aut}(M)$ by

$$
s_{\delta}(v):=v-\varepsilon\langle v, \delta\rangle \delta
$$

for all $v \in M$. Then $s_{\delta}$ is a reflection in the symmetric case and a symplectic transvection in the skew symmetric case. Let $\Gamma_{\Lambda} \subset \operatorname{Aut}(M)$ be the subgroup of $\operatorname{Aut}(M)$ generated by the transformations $s_{\delta}, \delta \in \Lambda$.

Definition. The pair $(M, \Lambda)$ is called a vanishing lattice, if it satisfies the following conditions:

(i) $\Lambda$ generates $M$. 
(ii) $\Lambda$ is an orbit of $\Gamma_{\Lambda}$ in $M$.

(iii) If $\operatorname{rank} M>1$, then there exist $\delta, \delta^{\prime} \in \Lambda$ such that $\left\langle\delta, \delta^{\prime}\right\rangle=1$.

It follows from Corollary 20 that, if it is not true that both $n$ is odd and 0 is a non-degenerate critical point of $f$, then the pair $\left(\widetilde{H}_{n}\left(X_{s}\right), \Lambda^{*}\right)$ is a vanishing lattice with $\varepsilon=(-1)^{n(n-1) / 2}$ and $\Gamma$ is the corresponding monodromy group.

We introduce some more algebraic notions. Let $M^{\#}=\operatorname{Hom}(M, \mathbb{Z})$ be the dual module and $j: M \rightarrow M^{\#}$ be the canonical homomorphism. A homomorphism $h: M \rightarrow M$ induces a homomorphism $h^{t}: M^{\#} \rightarrow M^{\#}$ of the dual modules. If $h$ leaves the bilinear form $\langle$,$\rangle invariant, then h^{t}(j(M)) \subset j(M)$. An automorphism $h \in \operatorname{Aut}(M)$ thus induces a homomorphism $h^{t}: M^{\#} / j(M) \rightarrow M^{\#} / j(M)$. Let Aut $^{\#}(M) \subset \operatorname{Aut}(M)$ be the subgroup of those automorphisms $h \in$ $\operatorname{Aut}(M)$ with $h^{t}=\operatorname{id}_{M \# / j(M)}$.

Now let $M$ be a skew symmetric lattice. It has a basis

$$
\left(e_{1}, f_{1}, \ldots, e_{m}, f_{m}, g_{1}, \ldots, g_{k}\right)
$$

such that

$$
\left\langle e_{i}, f_{i}\right\rangle=-\left\langle f_{i}, e_{i}\right\rangle=d_{i} \text { for } d_{i} \in \mathbb{Z}, d_{i} \geq 1, i=1, \ldots m,
$$

all other inner products are equal to zero, and $d_{i+1}$ is divisible by $d_{i}$ for $i=1, \ldots, m-1$. Such a basis is called a symplectic basis.

Let $\left(e_{1}, f_{1}, \ldots, e_{m}, f_{m}, g_{1}, \ldots, g_{k}\right)$ be a symplectic basis of $M$. Let $\eta_{2}$ be the exponent of 2 in the prime factor decomposition of $d_{m}$. Let $\mu=2 m+k$. We identify $M$ with $\mathbb{Z}^{\mu}$ through the symplectic basis $\left(e_{1}, f_{1}, \ldots, e_{m}, f_{m}, g_{1}, \ldots, g_{k}\right)$. A subgroup $G \subset \operatorname{Aut}^{\#}(M)$ then corresponds to a subgroup $\rho(G) \subset \operatorname{Sp}^{\#}(\mu, \mathbb{Z})$, where $\operatorname{Sp}^{\#}(\mu, \mathbb{Z})$ is the corresponding subgroup of the symplectic group

$$
\operatorname{Sp}(\mu, \mathbb{Z})=\left\{A \in \mathrm{GL}(\mu, \mathbb{Z}) \mid A^{t} J A=J\right\} .
$$

Let $r \in \mathbb{N} \backslash\{0\}$. A subgroup $G \subset \operatorname{Aut}^{\#}(M)$ is called a congruence subgroup modulo $r$ if

$$
\rho(G)=\left\{A \in \mathrm{Sp}^{\#}(\mu, \mathbb{Z}) \mid A \equiv E \bmod r\right\} .
$$

Here $E$ is the unit matrix and $A \equiv E \bmod r$ means that $a_{i j} \equiv \delta_{i j} \bmod r$ for all $1 \leq i, j \leq \mu$, where $A=\left(a_{i j}\right)$.

A congruence subgroup is obviously of finite index in the group $\operatorname{Aut}^{\#}(M)=\mathrm{Sp}^{\#}(M)$.

The following theorem was proved by W. A. M. Janssen [Jan83] based on previous work of A'Campo [A'C79], B. Wajnryb [Wa80], and S. V. Chmutov [Chm82, Chm83]. The notation $\langle v, M\rangle=\mathbb{Z}$ means that 
there is a $y \in M$ with $\langle v, y\rangle=1$. We write $a \in \Lambda \bmod 2$ if there is an element $b \in M$ with $a-2 b \in \Lambda$.

Theorem 31 (Janssen). Let $(M, \Lambda)$ be a skew symmetric vanishing lattice. Then

(i) $\Gamma_{\Lambda}$ contains the congruence subgroup modulo $2^{\eta_{2}+1}$ of $\mathrm{Sp}^{\#}(M)$,

(ii) $\Lambda=\{v \in M \mid\langle v, M\rangle=\mathbb{Z}$ and $v \in \Lambda \bmod 2\}$.

As a corollary, we get the following result:

Corollary 32. Let $f:\left(\mathbb{C}^{n+1}, 0\right) \rightarrow(\mathbb{C}, 0)$ be a holomorphic function germ with an isolated singularity at 0 and let $n$ be odd. Then

(i) $\Gamma$ contains the congruence subgroup modulo $2^{\eta_{2}+1}$ of $\mathrm{Sp}^{\#}(M)$,

(ii) $\Lambda^{*}=\left\{v \in M \mid\langle v, M\rangle=\mathbb{Z}\right.$ and $\left.v \in \Lambda^{*} \bmod 2\right\}$.

In (ii) it is assumed that 0 is not a non-degenerate critical point of $f$.

Janssen also proved a version of Theorem 31 for skew symmetric vanishing lattices over the field $\mathbb{F}_{2}$ and classified skew symmetric vanishing lattices over $\mathbb{F}_{2}$ [Jan83] and over $\mathbb{Z}$ [Jan85]. B. Shapiro, M. Shapiro, and A. Vainshtein [SSV98] applied these results to certain enumeration problems.

Now let $M$ be symmetric and let $\varepsilon \in\{-1,+1\}$. We put

$$
\bar{M}:=M / \operatorname{ker} j, \quad \bar{M}_{\mathbb{R}}:=\bar{M} \otimes \mathbb{R} .
$$

Then $\bar{M}_{\mathbb{R}}$ is a finite-dimensional real vector space with a nondegenerate symmetric bilinear form. Let $h \in \operatorname{Aut}(M)$ and $\bar{h}$ the induced element in $O\left(\bar{M}_{\mathbb{R}}\right)$. The transformation $\bar{h}$ can be written as a product of reflections

$$
\bar{h}=s_{v_{1}} \circ \ldots \circ s_{v_{r}}
$$

with $v_{i} \in \bar{M}_{\mathbb{R}},\left\langle v_{i}, v_{i}\right\rangle \neq 0, i=1, \ldots, r$. We define

$$
\nu_{\varepsilon}(h):= \begin{cases}+1 & \text { if } \varepsilon\left\langle v_{i}, v_{i}\right\rangle<0 \text { for an even number of indices, } \\ -1 & \text { otherwise. }\end{cases}
$$

The homomorphism $\nu_{\varepsilon}: \operatorname{Aut}(M) \rightarrow\{-1,+1\}$ is called the real $\varepsilon$-spinor norm.

Definition. We define a subgroup $O_{\varepsilon}^{*}(M) \subset O(M)$ as follows:

$$
O_{\varepsilon}^{*}(M):=\operatorname{Aut}^{\#}(M) \cap \operatorname{ker} \nu_{\varepsilon} .
$$

If $M$ is non-degenerate, then $M^{\#} / j(M)$ is a finite group and hence $O^{\#}(M)=\operatorname{Aut}^{\#}(M)$ is a subgroup of finite index in $O(M)=\operatorname{Aut}(M)$. The subgroup $\operatorname{ker} \nu_{\varepsilon} \subset O(M)$ is of index $\leq 2$ in $O(M)$. Thus if $M$ is non-degenerate, then $O_{\varepsilon}^{*}(M)$ is a subgroup of finite index in $O(M)$. 
Using a result of $\mathrm{M}$. Kneser [Kn81], the author proved the following theorem [Eb84]. A unimodular hyperbolic plane is a two-dimensional lattice with the bilinear form given by

$$
\left(\begin{array}{ll}
0 & 1 \\
1 & 0
\end{array}\right)
$$

Theorem 33. Let $(M, \Lambda)$ be an even symmetric vanishing lattice. Assume that $M$ contains a six-dimensional sublattice $K \subset M$ which is the orthogonal direct sum of two unimodular hyperbolic planes and a lattice of type $\varepsilon A_{2}$. Assume moreover $\{v \in K \mid\langle v, v\rangle=2 \varepsilon\} \subset \Lambda$. Then

(i) $\Gamma_{\Lambda}=O_{\varepsilon}^{*}(M)$,

(ii) $\Lambda=\{v \in M \mid\langle v, v\rangle=2 \varepsilon$ and $\langle v, M\rangle=\mathbb{Z}\}$.

From Theorem 33 one can derive the following theorem [Eb84]. The statement for the exceptional unimodal singularities was already proven by H. Pinkham [Pi77] (see also [Eb83a] for the history of the problem and previous results). It was noticed by Looijenga that (ii) is a consequence of (i).

Theorem 34. Let $f:\left(\mathbb{C}^{n+1}, 0\right) \rightarrow(\mathbb{C}, 0)$ be a holomorphic function germ with an isolated singularity at 0 and let $n$ be even, $\varepsilon=$ $(-1)^{n(n-1) / 2}$. Suppose that $f$ is not of type $T_{p, q, r}$ with $\frac{1}{p}+\frac{1}{q}+\frac{1}{r}<1$ and $(p, q, r) \neq(2,3,7),(2,4,5),(3,3,4)$. Then

(i) $\Gamma=O_{\varepsilon}^{*}(M)$,

(ii) $\Lambda^{*}=\{v \in M \mid\langle v, v\rangle=2 \varepsilon$ and $\langle v, M\rangle=\mathbb{Z}\}$.

In (ii) it is assumed that 0 is not a non-degenerate critical point of $f$.

Remark 35. Theorem 34 follows for the simple and simple elliptic singularities by the results stated in Sect. 6. It is false for the singularities of type $T_{p, q, r}$ with $\frac{1}{p}+\frac{1}{q}+\frac{1}{r}<1$ and $(p, q, r) \neq(2,3,7),(2,4,5),(3,3,4)$, see $[\mathrm{Eb} 81, \S 3]$. This follows from the fact that the graph of Fig. 9 with the vertex $\delta_{2}$ removed and with $\frac{1}{p}+\frac{1}{q}+\frac{1}{r}<1$ defines a Coxeter system of hyperbolic type if and only if $(p, q, r)=(2,3,7),(2,4,5),(3,3,4)$ [Bou02, Ch. V, $\S 4$, Exercice 12]. The three singularities $T_{p, q, r}$ with these values of $(p, q, r)$ are the minimal hyperbolic singularities. Theorem 34 was proved for these singularities by Brieskorn ([Br81, Theorem 2], but no proof is given). A proof following Brieskorn's proof can be found in [Eb87, 5.5]. A'Campo (unpublished) and Looijenga showed that the monodromy groups of these singularities have exponential growth. Looijenga's proof is published in [Du79, Appendix II]. 


\section{TOPOlogical EQUivalence}

We shall now consider the question to which extent the invariants determine the topological type of the singularity.

The topological type of a singularity $f:\left(\mathbb{C}^{n+1}, 0\right) \rightarrow(\mathbb{C}, 0)$ is described by the (local) embedding of the variety $f^{-1}(0)$ in a neighborhood of the singular point $0 \in \mathbb{C}^{n+1}$.

Definition. Two singularities $f, g:\left(\mathbb{C}^{n+1}, 0\right) \rightarrow(\mathbb{C}, 0)$ are topologically equivalent if there is a homeomorphism of neighborhoods $U$ and $V$ of the origin which maps $f^{-1}(0) \cap U$ to $g^{-1} \cap V$.

By [Mi68a, Theorem 2.10], the variety $f^{-1}(0)$ is locally the cone over its link $K$. By [Mi68a], the link is a fibered knot. A. Durfee [Du74] proved the following theorem.

Theorem 36 (Durfee). Let $n \geq 3$. There is a one-to-one correspondence of isotopy classes of fibered knots in $S^{2 n+1}$ and equivalence classes of integral unimodular bilinear forms given by associating to each fibered knot its Seifert form.

In view of the preceding remarks and Theorem 8 we obtain the following corollary.

Corollary 37. For $n \neq 2$ the set $\mathcal{D}$ of $f:\left(\mathbb{C}^{n+1}, 0\right) \rightarrow(\mathbb{C}, 0)$ determines $f$ up to topological equivalence.

This corollary was also proved by S. Szczepanski [Sz87]. Moreover, she showed in [Sz89] the following theorem.

Theorem 38 (Szczepanski). Two singularities $f, g:\left(\mathbb{C}^{3}, 0\right) \rightarrow(\mathbb{C}, 0)$ are topologically equivalent if

(i) the singularities have a common Coxeter-Dynkin diagram with respect to distinguished bases, and

(ii) the Milnor fibers have homeomorphic boundaries and the algebraic isomorphism of the Milnor lattices induced by the common Coxeter-Dynkin diagram is realized geometrically by either an inclusion of one Milnor fiber into the other or a homotopy equivalence of the Milnor fibers which induces a homeomorphism of the boundaries.

There is also the notion of $\mu$-homotopy or $\mu$-equivalence (see [Br88]).

Definition. Two singularities $f_{0}, f_{1}:\left(\mathbb{C}^{n+1}, 0\right) \rightarrow(\mathbb{C}, 0)$ are $\mu$-equivalent if there is a family $f_{t}:\left(\mathbb{C}^{n+1}, 0\right) \rightarrow(\mathbb{C}, 0)$ of analytic function germs with isolated singularities at the origin continuously depending on the parameter $t \in[0,1]$ with constant Milnor number $\mu\left(f_{t}\right)$. 
Lê Dũng Tráng and C. P. Ramanujam [LR76] proved the following theorem.

Theorem 39 (Lê-Ramanujam). If $n \neq 2$, then $\mu$-equivalent singularities are topologically equivalent.

The following proposition was proved by Gabrielov [Ga74b, Proposition 1].

Proposition 40 (Gabrielov). For two $\mu$-equivalent singularities there exist distinguished bases whose Coxeter-Dynkin diagrams coincide.

Using this proposition, one obtains the Lê-Ramanujam theorem as a consequence of Corollary 37. Moreover, one can derive from Theorem 38 a Lê-Ramanujam theorem for $n=2$, see [Sz89].

Now let $f:\left(\mathbb{C}^{n+1}, 0\right) \rightarrow(\mathbb{C}, 0)$ be an isolated singularity satisfying the conditions of Theorem 34. It follows from Theorem 34 that the invariants $\Gamma$ and $\Lambda^{*}$ are completely determined by $M$. The author [Eb81] has found examples of pairs of singularities (e.g. the bimodal singularities $Z_{17}$ and $Q_{17}$ in Arnold's notation) which have the same Coxeter-Dynkin diagrams with respect to weakly distinguished bases and the invariants $M, \Gamma$, and $\Lambda^{*}$ are the same, but the invariants $\mathcal{B}^{*}$ and $\mathcal{D}^{*}$ are different, the classical monodromy operators are not conjugate to each other, and the singularities are not topologically equivalent.

We conclude the article with some open questions which were posed to the author by late Brieskorn (around 1982?). We keep the condition that $f:\left(\mathbb{C}^{n+1}, 0\right) \rightarrow(\mathbb{C}, 0)$ is a singularity satisfying the conditions of Theorem 34. Let $n \equiv 2 \bmod 4$ and let $\mu$ be the Milnor number of $f$.

Problem 41 (Brieskorn). Let $M$ be the Milnor lattice (of rank $\mu$ ) and $\Gamma$ be the monodromy group of $f$. Let

$$
\Lambda:=\{v \in M \mid\langle v, v\rangle=-2\} .
$$

Then $\Gamma$ acts on $\Lambda$. Are there only finitely many orbits?

Problem 42 (Brieskorn). Let

$$
\mathcal{B}_{0}:=\left\{\left(e_{1}, \ldots, e_{\mu}\right) \in \Lambda^{\mu} \mid\left\langle e_{1}, \ldots, e_{\mu}\right\rangle_{\mathbb{Z}}=M\right\} .
$$

Then the group $\operatorname{Br}_{\mu}^{\rtimes}$ acts on $\mathcal{B}_{0}$. Are there only finitely many orbits? Alternatively, one can consider the set

$$
\widetilde{\mathcal{B}}_{0}:=\left\{\left(e_{1}, \ldots, e_{\mu}\right) \in\left(\Lambda^{*}\right)^{\mu} \mid\left\langle e_{1}, \ldots, e_{\mu}\right\rangle_{\mathbb{Z}}=M\right\}
$$

Problem 43 (Brieskorn). The group $\Gamma$ acts on $\mathcal{B}_{0}$ (or $\widetilde{\mathcal{B}}_{0}$ ) by

$$
\gamma\left(e_{1}, \ldots, e_{\mu}\right)=\left(\gamma e_{1}, \ldots, \gamma e_{\mu}\right) \text {. }
$$


This action commutes with the action of $\mathrm{Br}_{\mu}^{\rtimes}$. Are there only finitely many $\Gamma$-equivalence classes of $\mathrm{Br}_{\mu}^{\rtimes}$-orbits?

Very little is known about these problems. The answers to these questions are trivially yes for the simple singularities, since the sets $\Lambda$, $\mathcal{B}_{0}$, and $\widetilde{\mathcal{B}}_{0}$ are finite in this case. We have $\Lambda=\Lambda^{*}$ (and hence there is only one $\Gamma$-orbit) for the simple, simple elliptic, and minimal hyperbolic singularities (for the latter ones see [Eb87, Proposition 5.5.1]).

An element $c \in \Gamma$ for which there exists a basis $\left(e_{1}, \ldots, e_{\mu}\right) \in \mathcal{B}_{0}$ such that $c=s_{e_{1}} \cdots s_{e_{\mu}}$ is called a quasi Coxeter element.

Problem 44 (Brieskorn). Let $c \in \Gamma$ be a quasi Coxeter element and let

$$
\mathcal{B}_{0, c}:=\left\{\left(e_{1}, \ldots, e_{\mu}\right) \in \mathcal{B}_{0} \mid s_{e_{1}} \cdots s_{e_{\mu}}=c\right\} .
$$

The set $\mathcal{B}_{0, c}$ is invariant under the action of the group $\operatorname{Br}_{\mu}^{\rtimes}$. What is the relation between the orbits of $\mathrm{Br}_{\mu}^{\rtimes}$ on $\mathcal{B}_{0}$ and the sets $\mathcal{B}_{0, c}$ ?

For the simple singularities, the quasi Coxeter elements were determined up to conjugacy by E. Voigt [Vo85a, Vo85b] and he showed that the group $\mathrm{Br}_{\mu}^{\rtimes}$ acts transitively on $\mathcal{B}_{0, c}$ for each quasi Coxeter element $c$. For $c$ being the classical monodromy operator, it is known for the simple (Corollary 27), the simple elliptic [Kl86], and the hyperbolic singularities [BWY19] that the group $\mathrm{Br}_{\mu}^{\rtimes}$ acts transitively on $\mathcal{B}_{0, c}$ (see Sect. 6 above). To the author's knowledge, this is all what is known about Problem 44.

\section{REFERENCES}

[A'C73] N. A'Campo: Le nombre de Lefschetz d'une monodromie. Indag. Math. 35 (1973), 113-118.

[A'C75a] N. A'Campo: Le groupe de monodromie du déploiement des singularités isolées de courbes planes. I. Math. Ann. 213 (1975), 1-32.

[A'C75b] N. A'Campo: Le groupe de monodromie du déploiement des singularités isolées de courbes planes. II. Proceedings of the International Congress of Mathematicians (Vancouver, B. C., 1974), Vol. 1, pp. 395404. Canad. Math. Congress, Montreal, Que., 1975.

[A'C75c] N. A'Campo: La fonction zêta d'une monodromie. Comment. Math. Helv. 50 (1975), 233-248.

[A'C76] N. A'Campo: Sur les valeurs propres de la transformation de Coxeter. Invent. Math. 33 (1976), no. 1, 61-67.

[A'C79] N. A'Campo: Tresses, monodromie et le groupe symplectique. Comment. Math. Helv. 54 (1979), no. 2, 318-327.

[AT19] D. Aramaki, A. Takahashi: Maximally-graded matrix factorizations for an invertible polynomial of chain type. Preprint arXiv:1903.02732.

[Arn72] V. I. Arnold: Normal forms of functions near degenerate critical points, the Weyl groups $A_{k}, D_{k}, E_{k}$ and Lagrangian singularities. Funktsional. 
Anal. i Prilozen. 6 (1972), no. 4, 3-25 (Engl. translation in Funct. Anal. Appl. 6 (1972), no. 4, 254-272).

[Arn73] V. I. Arnold: Remarks on the method of stationary phase and on the Coxeter numbers. Usp. Mat. Nauk 28:5 (1973), 17-44 (Engl. translation in Russ. Math. Surv. 28:5 (1973), 19-48).

[Arn75] V. I. Arnold: Critical points of smooth functions and their normal forms. Usp. Mat. Nauk. 30:5 (1975), 3-65 (Engl. translation in Russ. Math. Surv. 30:5 (1975), 1-75).

[Arn96] V. I. Arnold: Topological classification of complex trigonometric polynomials and the combinatorics of graphs with an identical number of vertices and edges. Funktsional. Anal. i Prilozhen. 30 (1996), no. 1, 1-17 (Engl. translation in Funct. Anal. Appl. 30 (1996), no. 1, 1-14).

[AGV85] V. I. Arnold, S. M. Gusein-Zade, A. N.Varchenko: Singularities of Differentiable Maps, Volume I. Birkhäuser, Boston-Basel-Berlin, 1985.

[AGV88] V. I. Arnold, S. M. Gusein-Zade, A. N.Varchenko: Singularities of Differentiable Maps, Volume II. Birkhäuser, Boston-Basel-Berlin, 1988.

[Art25] E. Artin: Theorie der Zöpfe. Abh. Math. Sem. Univ. Hamburg 4 (1925), $101-126$.

[Art47] E. Artin: Theory of braids. Ann. of Math. (2) 48 (1947), 101-126.

[BK91] D. Bättig, H. Knörrer: Singularitäten. Birkhäuser-Verlag, Basel, 1991.

[BDSW14] B. Baumeister, M. Dyer, Ch. Stump, P. Wegener: A note on the transitive Hurwitz action on decompositions of parabolic Coxeter elements. Proc. Amer. Math. Soc. Ser. B 1 (2014), 149-154.

[BGRW17] B. Baumeister, Th. Gobet, K. Roberts, P. Wegener: On the Hurwitz action in finite Coxeter groups. J. Group Theory 20 (2017), no. 1, 103131.

[BWY19] B. Baumeister, P. Wegener, S. Yahiatene: Extended Weyl groups and Hurwitz transitivity. Preprint 2019.

[Bes03] D. Bessis: The dual braid monoid. Ann. Sci. École Norm. Sup. (4) 36 (2003), no. 5, 647-683.

[Bes15] D. Bessis: Finite complex reflection arrangements are $K(\pi, 1)$. Ann. of Math. (2) 181 (2015), no. 3, 809-904.

[Bey72a] C. H. Bey: Sur l'irréductibilité de la monodromie locale. C. R. Acad. Sci. Paris Sér. A-B 275 (1972), A21-A24.

[Bey72b] C. H. Bey: Sur l'irréductibilité de la monodromie locale; application à l'équisingularité. C. R. Acad. Sci. Paris Sér. A-B 275 (1972), A105A107.

[Bir74] J. S. Birman: Braids, links, and mapping class groups. Annals of Mathematics Studies, No. 82. Princeton University Press, Princeton, N.J.; University of Tokyo Press, Tokyo, 1974.

[Bou02] N. Bourbaki: Lie groups and Lie algebras. Chapters 4-6. Translated from the 1968 French original by Andrew Pressley. Elements of Mathematics (Berlin). Springer-Verlag, Berlin, 2002.

[Br66] E. Brieskorn: Beispiele zur Differentialtopologie von Singularitäten. Invent. Math. 2 (1966), 1-14.

[Br70] E. Brieskorn: Die Monodromie der isolierten Singularitäten von Hyperflächen. Manuscripta math. 2 (1970), 103-160. 
[Br71] E. Brieskorn: Die Fundamentalgruppe des Raumes der regulären Orbits einer endlichen komplexen Spiegelungsgruppe. Invent. Math. 12 (1971), $57-61$.

[Br73a] E. Brieskorn: Sur les groupes de tresses [d'après V. I. Arnol'd]. Séminaire Bourbaki, 24me année (1971/1972), Exp. No. 401, pp. 21-44. Lecture Notes in Math., Vol. 317, Springer, Berlin, 1973.

[Br73b] E. Brieskorn: Vue d'ensemble sur les problèmes de monodromie. Singularités à Cargèse, (Rencontre sur les Singularités en Géométrie Analytique, Inst. Études Sci. de Cargèse, 1972), pp. 393-413. Astérisque Nos. 7 et 8, Soc. Math. France, Paris, 1973.

[Br81] E. Brieskorn: The unfolding of exceptional singularities. Leopoldina Symposium: Singularities (Thüringen, 1978). Nova Acta Leopoldina (N.F.) 52 (1981), no. 240, 65-93.

[Br83] E. Brieskorn: Milnor lattices and Dynkin diagrams. Singularities, Part 1 (Arcata, Calif., 1981), 153-165, Proc. Sympos. Pure Math., 40, Amer. Math. Soc., Providence, RI, 1983.

[Br88] E. Brieskorn: Automorphic sets and braids and singularities. Braids (Santa Cruz, CA, 1986), 45-115, Contemp. Math., 78, Amer. Math. Soc., Providence, RI, 1988.

[BS72] E. Brieskorn, K. Saito: Artin-Gruppen und Coxeter-Gruppen. Invent. Math. 17 (1972), 245-271.

[By15] B. S. Bychkov: On decompositions of a cyclic permutation into a product of a given number of permutations. Funktsional. Anal. i Prilozhen. 49 (2015), no. 2, 1-6. (Engl. translation in Funct. Anal. Appl. 49 (2015), no. $2,81-85)$.

[CV93] S. Cecotti, C. Vafa: On classification of $\mathrm{N}=2$ supersymmetric theories. Comm. Math. Phys. 158 (1993), no. 3, 569-644.

[Chm82] S. V. Chmutov: Monodromy groups of critical point of functions. Invent. Math. 67 (1982), no. 1, 123-131.

[Chm83] S. V. Chmutov: The monodromy groups of critical points of functions. II. Invent. Math. 73 (1983), no. 3, 491-510.

[Co89] A. J. Coleman: Killing and the Coxeter transformation of Kac-Moody algebras. Invent. Math. 95 (1989), no. 3, 447-477.

[CB93] W. Crawley-Boevey: Exceptional sequences of representations of quivers. Representations of algebras (Ottawa, ON, 1992), 117-124, CMS Conf. Proc., 14, Amer. Math. Soc., Providence, RI, 1993.

[Deh00] P. Dehornoy: Braids and self-distributivity. Progress in Mathematics, 192. Birkhäuser Verlag, Basel, 2000.

[Del72] P. Deligne: Les immeubles des groupes de tresses généralisés. Invent. Math. 17 (1972), 273-302.

[Del74] P. Deligne: Letter to Looijenga on March 9, 1974 (French). Available at https://homepage.rub.de/christian.stump/Deligne_Looijenga_Letter_09-03-1974.pdf.

[DK73] P. Deligne, N. Katz: Groupes de monodromie en géométrie algébrique. II. Séminaire de Géométrie Algébrique du Bois-Marie 1967-1969 (SGA 7 II). Lecture Notes in Mathematics, Vol. 340. Springer-Verlag, BerlinNew York, 1973. 
[Dem75] M. Demazure: Classification des germes à point critique isolé et à nombres de modules 0 ou 1 (d'après V. I. Arnold). Séminaire Bourbaki, Vol. 1973/1974, 26ème année, Exp. No. 443, pp. 124-142. Lecture Notes in Math., Vol. 431, Springer, Berlin, 1975.

[Di06] F. Digne: Présentations duales des groupes de tresses de type affine $\widetilde{A}$. Comment. Math. Helv. 81 (2006), no. 1, 23-47.

[Du74] A. H. Durfee: Fibered knots and algebraic singularities. Topology 13 (1974), 47-59.

[Du79] A. H. Durfee: Fifteen characterizations of rational double points and simple critical points. Enseign. Math. (2) 25 (1979), no. 1-2, 131-163.

[Eb80] W. Ebeling: Quadratische Formen und Monodromiegruppen von Singularitäten. Dissertation, Rheinische Friedrich-Wilhelms-Universität, Bonn, 1980.

[Eb81] W. Ebeling: Quadratische Formen und Monodromiegruppen von Singularitäten. Math. Ann. 255 (1981), 463-498.

[Eb83a] W. Ebeling: On the monodromy groups of singularities. Singularities, Part 1 (Arcata, Calif., 1981), 327-336, Proc. Sympos. Pure Math., 40, Amer. Math. Soc., Providence, RI, 1983.

[Eb83b] W. Ebeling: Milnor lattices and geometric bases of some special singularities. Nouds, tresses et singularités (Ed. C.Weber), Monographie Enseign. Math. 31, Genève, 1983, 129-146 and Enseign. Math. (2) 29 (1983), 263-280.

[Eb84] W. Ebeling: An arithmetic characterisation of the symmetric monodromy groups of singularities. Invent. Math. 77 (1984), no. 1, 85-99.

[Eb87] W. Ebeling: The Monodromy Groups of Isolated Singularities of Complete Intersections. Lect. Notes in Math., Vol. 1293, Springer-Verlag, Berlin etc., 1987.

[Eb96] W. Ebeling: On Coxeter-Dynkin diagrams of hypersurface singularities. J. Math. Sciences 82 (1996), 3657-3664.

[Eb06] W. Ebeling: Monodromy. Singularities and computer algebra, 129-155, London Math. Soc. Lecture Note Ser., 324, Cambridge Univ. Press, Cambridge, 2006.

[Eb07] W. Ebeling: Functions of several complex variables and their singularities. Graduate Studies in Math. Vol. 83, American Mathematical Society, Providence R. I., 2007.

[Eb17] W. Ebeling: Homological mirror symmetry for singularities. Representation theory - current trends and perspectives, 75-107, EMS Ser. Congr. Rep., Eur. Math. Soc., Zürich, 2017.

[Eb18] W. Ebeling: A note on distinguished bases of singularities. Topology Appl. 234 (2018), 259-268.

[EG07] W. Ebeling, S. M. Gusein-Zade: Lectures on monodromy. Singularities in geometry and topology, 234-252, World Sci. Publ., Hackensack, NJ, 2007.

[EP13] W. Ebeling, D. Ploog: A geometric construction of Coxeter-Dynkin diagrams of bimodal singularities. Manuscripta Math. 140 (2013), no. $1-2,195-212$. 
[FHHI03] B. Feng, A. Hanany, Y. He, A. Iqbal: Quiver theories, soliton spectra and Picard-Lefschetz transformations. J. High Energy Phys. 2003, no. 2, 056, 33 pp.

[FH03] S. Franco, A. Hanany: Toric duality, Seiberg duality and PicardLefschetz transformations. Proceedings of the 35th International Symposium Ahrenshoop on the Theory of Elementary Particles (BerlinSchmöckwitz, 2002). Fortschr. Phys. 51 (2003), no. 7-8, 738-744.

[Ga73] A. M. Gabrielov: Intersection matrices for certain singularities. Funktsional. Anal. i Prilozen. 7 (1973), no. 3, 18-32. (Engl. translation in Funct. Anal. Appl. 7 (1974), no. 3, 182-193).

[Ga74a] A. M. Gabrielov: Bifurcations, Dynkin diagrams and the modality of isolated singularities. Funktsional. Anal. i Prilozen. 8 (1974), no. 2, 7 12. (Engl. translation in Funct. Anal. Appl. 8 (1974), no. 2, 94-98).

[Ga74b] A. M. Gabrielov: Dynkin diagrams of unimodal singularities. Funktsional. Anal. i Prilozen. 8 (1974), no. 3, 1-6 (Engl. translation in Funct. Anal. Appl. 8 (1974), no. 3, 192-196).

[Ga79] A. M. Gabrielov: Polar curves and intersection matrices of singularities. Invent. math. 54 (1979), 15-22.

[Gi88] A. B. Givental: Twisted Picard-Lefschetz formulas. Funktsional. Anal. i Prilozhen. 22 (1988), no. 1, 12-22 (Engl. translation in Funct. Anal. Appl. 22 (1988), no. 1, 10-18).

[Gr19] G.-M. Greuel: Deformation and smoothing of singularities. This handbook.

[GZ74] S. M. Gusein-Zade: Intersection matrices for certain singularities of functions of two variables. Funktsional. Anal. i Prilozen. 8 (1974), no. 1, 1-15 (Engl. translation in Funct. Anal. Appl. 8 (1974), no. 1, 10-13).

[GZ75] S. M. Gusein-Zade: Dynkin diagrams of the singularities of functions of two variables. Funktsional. Anal. i Prilozen. 8 (1974), no. 4, 23-30 (Engl. translation in Funct. Anal. Appl. 8 (1975), no. 4, 295-300).

[GZ77] S. M. Gusein-Zade: Monodromy groups of isolated singularities of hypersurfaces. Uspehi Mat. Nauk 32 (1977), no. 2, 23-65 (Engl. translation in Russian Math. Surveys 32 (1977), no. 2, 23-69).

[GZ80] S. M. Gusein-Zade: Distinguished bases of simple singularities. Funktsional. Anal. i Prilozhen. 14 (1980), no. 4, 73-74 (Engl. translation in Funct. Anal. Appl. 14 (1980), no. 4, 307-308).

[HL74] A. Hefez, F. Lazzeri: The intersection matrix of Brieskorn singularities. Invent. Math. 25 (1974), 143-157.

[HR18] C. Hertling, C. Roucairol: Distinguished bases and Stokes regions for the simple and the simple elliptic singularities. Preprint arXiv: 1806.00996.

[HM68] F. Hirzebruch, K. H. Mayer: $\mathrm{O}(n)$-Mannigfaltigkeiten, exotische Sphären und Singularitäten. Lecture Notes in Mathematics, No. 57 Springer-Verlag, Berlin-New York 1968 iv+132 pp.

[HK16] A. Hubery, H. Krause: A categorification of non-crossing partitions. J. Eur. Math. Soc. (JEMS) 18 (2016), no. 10, 2273-2313.

[Hum85] St. Humphries: On weakly distinguished bases and free generating sets of free groups. Quart. J. Math. Oxford Ser. (2) 36 (1985), no. 142, $215-219$. 
[Hur91] A. Hurwitz: Ueber Riemann'sche Flächen mit gegebenen Verzweigungspunkten. Math. Ann. 39 (1891), no. 1, 1-60.

[Ig11] K. Igusa: Exceptional sequences, braid groups and clusters. Groups, algebras and applications, 227-240, Contemp. Math., 537, Amer. Math. Soc., Providence, RI, 2011.

[IS10] K. Igusa, R. Schiffler: Exceptional sequences and clusters. J. Algebra 323 (2010), no. 8, 2183-2202.

[Il87] G. G. Il'yuta: On the Coxeter transformation of an isolated singularity. Usp. Mat. Nauk 42 (1987), no. 2, 227-228 (Engl. translation in Russ. Math. Surveys 42 (1987), no. 2, 279-280).

[I194] G. G. Il'yuta: A'Campo's theorem on the discriminant. Funktsional. Anal. i Prilozen. 28 (1994), no. 2, 12-20 (Engl. translation in Funct. Anal. Appl. 28 (1994), no. 2, 85-91).

[I195] G. G. Il'yuta: Characterization of simple Coxeter-Dynkin diagrams. Funktsional. Anal. i Prilozen. 29 (1995), no. 3, 72-75 (Engl. translation in Funct. Anal. Appl. 29 (1995), no. 3, 205-207).

[I196] G. G. Il'yuta: On $q$-monodromy groups of singularities. Izv. Ross. Akad. Nauk Ser. Mat. 60 (1996), no. 1, 115-132 (Engl. translation in Izv. Math. 60 (1996), no. 1, 119-136).

[I197] G. G. Il'yuta: Coxeter-Dynkin diagrams of partially ordered sets and A'Campo-Gusein-Zade diagrams of simple singularities. Funktsional. Anal. i Prilozen. 31 (1997), no. 1, 12-19 (Engl. translation in Funct. Anal. Appl. 31 (1997), no. 1, 10-15).

[Jan83] W. A. M. Janssen: Skew-symmetric vanishing lattices and their monodromy groups. Math. Ann. 266 (1983), no. 1, 115-133.

[Jan85] W. A. M. Janssen: Skew-symmetric vanishing lattices and their monodromy groups. II. Math. Ann. 272 (1985), no. 1, 17-22.

[Jaw86] P. Jaworski: Distribution of critical values of miniversal deformations of parabolic singularities. Invent. Math. 86 (1986), no. 1, 19-33.

[Jaw88] P. Jaworski: Decompositions of parabolic singularities. Bull. Sci. Math. (2) 112 (1988), no. 2, 143-176.

[KY11] B. Keller, D. Yang: Derived equivalences from mutations of quivers with potential. Adv. Math 226 (2011), 2118-2168.

[Kl86] P. Kluitmann: Ausgezeichnete Basen erweiterter affiner Wurzelgitter. Dissertation, Rheinische Friedrich-Wilhelms-Universität, Bonn, 1986. Bonner Mathematische Schriften, 185. Universität Bonn, Mathematisches Institut, Bonn, 1987. vi+142 pp.

[Kl88] P. Kluitmann: Hurwitz action and finite quotients of braid groups. Braids (Santa Cruz, CA, 1986), 299-325, Contemp. Math., 78, Amer. Math. Soc., Providence, RI, 1988.

[K189] P. Kluitmann: Addendum zu der Arbeit: "Ausgezeichnete Basen von Milnorgittern einfacher Singularitäten" von E. Voigt. Abh. Math. Sem. Univ. Hamburg 59 (1989), 123-124.

[Kn81] M. Kneser: Erzeugung ganzzahliger orthogonaler Gruppen durch Spiegelungen. Math. Ann. 255 (1981), no. 4, 453-462.

[Lam75] K. Lamotke: Die Homologie isolierter Singularitäten. Math. Z. 143 (1975), 27-44. 
[Lam81] K. Lamotke: The topology of complex projective varieties after S. Lefschetz. Topology 20 (1981), no. 1, 15-51.

[Laz73] F. Lazzeri: A theorem on the monodromy of isolated singularities. Singularités à Cargèse (Rencontre Singularités Géom. Anal., Inst. Études Sci., Cargèse, 1972), pp. 269-275. Astérisque, Nos. 7 et 8, Soc. Math. France, Paris, 1973.

[Laz74] F. Lazzeri: Some remarks on the Picard-Lefschetz monodromy. Quelques journées singulières, i+9 pp. Centre Math. École Polytechnique, Paris, 1974.

[Le73] Lê Dũng Tráng: Topologie des singularités des hypersurfaces complexes. Singularités à Cargèse (Rencontre Singularités Géom. Anal., Inst. Études Sci., Cargèse, 1972), pp. 171-182. Astérisque, Nos. 7 et 8, Soc. Math. France, Paris, 1973.

[Le78] Lê Dũng Tráng: The geometry of the monodromy theorem. C. P. Ramanujam - a tribute, pp. 157-173, Tata Inst. Fund. Res. Studies in Math., 8, Springer, Berlin-New York, 1978.

[LR76] Lê Dũng Tráng, C. P. Ramanujam: The invariance of Milnor's number implies the invariance of the topological type. Amer. J. Math. 98 (1976), no. $1,67-78$.

[Lef24] S. Lefschetz: L'analysis situs et la géométrie algébrique. GauthierVillars, Paris, 1924.

[vdL83] H. van der Lek: Extended Artin groups. Singularities, Part 2 (Arcata, Calif., 1981), 117-121, Proc. Sympos. Pure Math., 40, Amer. Math. Soc., Providence, RI, 1983.

[Li81] I. S. Livshits: Automorphisms of the complement to the bifurcation set of functions for simple singularities. Funktsional. Anal. i Prilozhen. 15 (1981), no. 1, 38-42 (Engl. translation in Funct. Anal. Appl. 15 (1981), no. 1, 29-32).

[Loe07] M. Lönne: Fundamental group of discriminant complements of Brieskorn-Pham polynomials. C. R. Math. Acad. Sci. Paris 345 (2007), no. 2, 93-96.

[Loe10] M. Lönne: Braid monodromy of some Brieskorn-Pham singularities. Internat. J. Math. 21 (2010), no. 8, 1047-1070.

[Loo74] E. Looijenga: The complement of the bifurcation variety of a simple singularity. Invent. Math. 23 (1974), 105-116.

[Loo78] E. Looijenga: On the semi-universal deformation of a simple-elliptic hypersurface singularity. II. The discriminant. Topology 17 (1978), no. $1,23-40$.

[Loo80] E. Looijenga: Invariant theory for generalized root systems. Invent. Math. 61 (1980), no. 1, 1-32.

[Loo81] E. Looijenga: Rational surfaces with an anticanonical cycle. Ann. of Math. (2) 114 (1981), no. 2, 267-322.

[Loo84] E. Looijenga: Isolated singular points on complete intersections. London Mathematical Society Lecture Note Series, 77. Cambridge University Press, Cambridge, 1984.

[Ly79] O. V. Lyashko: The geometry of bifurcation diagrams. Uspekhi Mat. Nauk 34 (1979), no. 3, 205-206 (Engl. translation in Russian Math. Surveys 34 (1979), no. 3, 209-210). 
[Ly84] O. V. Lyashko: The geometry of bifurcation diagrams. J. Soviet Math. 27 (1984), 2736-2759.

[LS77] R. C. Lyndon, P. E. Schupp: Combinatorial group theory. Ergebnisse der Mathematik und ihrer Grenzgebiete, Band 89. Springer-Verlag, Berlin-New York, 1977.

[McC86] J. McCool: On basis-conjugating automorphisms of free groups. Canad. J. Math. 38 (1986), no. 6, 1525-1529.

[Mi68a] J. Milnor: Singular Points of Complex Hypersurfaces. Ann. of Math. Studies Vol. 61, Princeton University Press, Princeton, 1968.

[Mi68b] J. Milnor: A note on curvature and fundamental group. J. Differential Geometry 2 (1968), 1-7.

[OR77] P. Orlik, R. Randell: The monodromy of weighted homogeneous singularities. Invent. Math. 39 (1977), 199-211.

[Ph65] F. Pham: Formules de Picard-Lefschetz généralisées et ramification des intégrales. Bull. Soc. Math. France 93 (1965), 333-367.

[PS97] E. Picard, S. Simart: Traité des fonctions algébriques de deux variables. Vol. I. Gauthier-Villars, Paris, 1897.

[Pi77] H. Pinkham: Groupe de monodromie des singularités unimodulaires exceptionnelles. C. R. Acad. Sci. Paris Sér. A-B 284 (1977), no. 23, A1515-A1518.

[Rin94] C. M. Ringel: The braid group action on the set of exceptional sequences of a hereditary Artin algebra. Abelian group theory and related topics (Oberwolfach, 1993), 339-352, Contemp. Math., 171, Amer. Math. Soc., Providence, RI, 1994.

[Rip10] V. Ripoll: Orbites d'Hurwitz des factorisations primitives d'un élément de Coxeter. J. Algebra 323 (2010), no. 5, 1432-1453.

[Rip12] V. Ripoll: Lyashko-Looijenga morphisms and submaximal factorizations of a Coxeter element. J. Algebraic Combin. 36 (2012), no. 4, 649-673.

[Sa74] K. Saito: Einfach-elliptische Singularitäten. Invent. Math. 23 (1974), 289-325.

[Sa82] K. Saito: A characterization of the intersection form of a Milnor's fiber for a function with an isolated critical point. Proc. Japan Acad. Ser. A Math. Sci. 58 (1982), no. 2, 79-81.

[Sa85] K. Saito: Extended affine root systems. I. Coxeter transformations. Publ. Res. Inst. Math. Sci. 21 (1985), no. 1, 75-179.

[ST71] M. Sebastiani, R. Thom: Un résultat sur la monodromie. Invent. Math. 13 (1971), 90-96.

[Se01] H. Serizawa: Distinguished bases of non-simple singularities. Tokyo J. Math. 24 (2001), no. 1, 19-38.

[SSV98] B. Shapiro, M. Shapiro, A. Vainshtein: Skew-symmetric vanishing lattices and intersections of Schubert cells. Internat. Math. Res. Notices 1998, no. 11, 563-588.

[Si74] D. Siersma: Classification and deformation of singularities. Doctoral dissertation, University of Amsterdam. University of Amsterdam, Amsterdam, 1974. ii+115 pp. (1 foldout).

[Sz87] S. Szczepanski: Geometric bases and topological equivalence. Comm. Pure Appl. Math. 40 (1987), no. 3, 389-399. 
[Sz89] S. Szczepanski: Criteria for topological equivalence and a LêRamanujam theorem for three complex variables. Duke Math. J. 58 (1989), no. 2, 513-530.

[Ty68] G. N. Tyurina: The topological properties of isolated singularities of complex spaces of codimension one. Izv. Akad. Nauk SSSR Ser. Mat. 32 (1968), no. 3, 605-620 (Engl. translation in Math. USSR-Izv. 2:3 (1968), 557-571).

[Va15] V. A. Vassiliev: A few problems on monodromy and discriminants. Arnold Math. J. 1 (2015), no. 2, 201-209.

[Vo85a] E. Voigt: Ausgezeichnete Basen von Milnorgittern einfacher Singularitäten. Dissertation, Rheinische Friedrich-Wilhelms-Universität Bonn, Bonn, 1984. Bonner Mathematische Schriften, 160. Universität Bonn, Mathematisches Institut, Bonn, 1985. ii+150 pp.

[Vo85b] E. Voigt: Ausgezeichnete Basen von Milnorgittern einfacher Singularitäten. Abh. Math. Sem. Univ. Hamburg 55 (1985), 183-190.

[Wa80] B. Wajnryb: On the monodromy group of plane curve singularities. Math. Ann. 246 (1979/80), no. 2, 141-154.

[Yu96] Jianming Yu: Combinatorial structure of Stokes regions of a simple singularity. Math. Ann. 305 (1996), no. 2, 355-368.

[Yu99] Jianming Yu: Galois group of Looijenga-Lyashko mapping. Math. Z. 232 (1999), no. 2, 321-330.

[ZL99] D. Zvonkine, S. K. Lando: On multiplicities of the Lyashko-Looijenga mapping on strata of the discriminant. Funktsional. Anal. i Prilozhen. 33 (1999), no. 3, 21-34 (Engl. translation in Funct. Anal. Appl. 33 (1999), no. $3,178-188)$. 


\section{INDEX}

$O_{\varepsilon}^{*}(M), 33$

$\Lambda^{*}, 6$

$\alpha_{i}(j), 17$

$\alpha_{j}, 13$

$\beta_{i}(j), 17$

$\beta_{j+1}, 14$

$\mathcal{B}, 15$

$\mathcal{B}^{*}, 16$

$\mathcal{D}, 15$

$\mathcal{D}^{*}, 16$

$\kappa_{i}, 15$

$\mu$-equivalent, 35

$\mu=$ const stratum, 25

$\mathrm{Br}_{\mu}^{\rtimes}, 15$

automorphic set, 16

basis

distinguished, 9

geometric, 9, 16

symplectic, 32

weakly distinguished, 9

bifurcation variety, 24

bimodal singularities, 25

bimodular singularities, 25

Brieskorn-Pham singularities, 19

classical monodromy, 4

classical monodromy operator, 4

congruence subgroup, 32

corank, 24

Coxeter element, 11

quasi, 37

Coxeter-Dynkin diagram, 11

cycle

monotone, 30

vanishing, 6

diagram

Coxeter-Dynkin, 11

discriminant, 22

distinguished basis, 9

distinguished system, 8

element

Coxeter, 11

equivalent

right, 24 topologically, 35

fiber

Milnor, 4

fibration

Milnor, 4

form

Seifert, 12

geometric basis, 9, 16

geometric monodromy, 4

group

monodromy, 9

homotopic, 14

hyperbolic singularities, 28

minimal, 34

intersection matrix, 11

lattice

Milnor, 4

vanishing, 31

link, 12

loop

simple, 7

Lyashko-Looijenga mapping, 24

mapping

Lyashko-Looijenga, 24

matrix

intersection, 11

Milnor fiber, 4

Milnor fibration, 4

Milnor lattice, 4

minimal hyperbolic singularities, 34

modality, 24

module number, 24

monodromy

classical, 4

geometric, 4

monodromy group, 9

monotone cycle, 30

morsification, 4

operator

classical monodromy, 4

parabolic singularities, 27 
Picard-Lefschetz transformation, 7

quasi Coxeter element, 37

reflection, 7

right equivalent, 24

Sebastiani-Thom sum, 18

Seifert form, 12

set

automorphic, 16

simple elliptic singularities, 27

simple loop, 7

simple singularities, 25

singularities

bimodal, 25

bimodular, 25

Brieskorn-Pham, 19

hyperbolic, 28

minimal hyperbolic, 34

parabolic, 27

simple, 25

simple elliptic, 27

unimodal, 25

unimodular, 25

spinor norm, 33

stabilization, 19

sum

Sebastiani-Thom, 18

symplectic basis, 32

symplectic transvection, 7

system

distinguished, 8

weakly distinguished, 8

topologically equivalent, 35

transformation

Picard-Lefschetz, 7

transvection

symplectic, 7

unfolding, 4

unimodal singularities, 25

unimodular singularities, 25

vanishing cycle, 6

vanishing lattice, 31

variety

bifurcation, 24 weakly distinguished basis, 9 weakly distinguished system, 8 weight, 31 
Institut für Algebraische Geometrie, Leibniz Universität Hannover, Postfach 6009, D-30060 Hannover, Germany

E-mail address: ebeling@math.uni-hannover.de 